\title{
A New Kinetic Framework for Synaptic Vesicle Trafficking Tested in Synapsin Knock-Outs
}

\author{
Thomas Gabriel, ${ }^{1 \star}$ Elizabeth García-Pérez, ${ }^{1 *}$ Kashif Mahfooz, ${ }^{1}$ Joaquín Goñi, ${ }^{1,2}$ Rebeca Martínez-Turrillas, ${ }^{1}$ \\ Isabel Pérez-Otaño, ${ }^{1}$ Donald C. Lo, ${ }^{3}$ and John F. Wesseling ${ }^{1}$ \\ ${ }^{1}$ Departamento de Neurociencias, Centro de Investigación Médica Aplicada (CIMA), and ²Departamento de Física y Matemática Aplicada, Universidad de \\ Navarra, 31008 Pamplona, Spain, and ${ }^{3}$ Center for Drug Discovery and Department of Neurobiology, Duke University Medical Center, Durham, North \\ Carolina 27710
}

At least two rate-limiting mechanisms in vesicle trafficking operate at mouse Schaffer collateral synapses, but their molecular/physical identities are unknown. The first mechanism determines the baseline rate at which reserve vesicles are supplied to a readily releasable pool. The second causes the supply rate to depress threefold when synaptic transmission is driven hard for extended periods. Previous models invoked depletion of a reserve vesicle pool to explain the reductions in the supply rate, but the mass-action assumption at their core is not compatible with kinetic measurements of neurotransmission under maximal-use conditions. Here we develop a new theoretical model of rate-limiting steps in vesicle trafficking that is compatible with previous and new measurements. A physical interpretation is proposed where local reserve pools consisting of four vesicles are tethered to individual release sites and are replenished stochastically in an all-or-none fashion. We then show that the supply rate depresses more rapidly in synapsin knock-outs and that the phenotype can be fully explained by changing the value of the single parameter in the model that would specify the size of the local reserve pools. Vesicle-trafficking rates between pools were not affected. Finally, optical imaging experiments argue against alternative interpretations of the theoretical model where vesicle trafficking is inhibited without reserve pool depletion. This new conceptual framework will be useful for distinguishing which of the multiple molecular and cell biological mechanisms involved in vesicle trafficking are rate limiting at different levels of synaptic throughput and are thus candidates for physiological and pharmacological modulation.

\section{Introduction}

Short-term depression at Schaffer collateral synapses includes the serial emergence of two rate-limiting presynaptic vesicletrafficking mechanisms (Garcia-Perez et al., 2008). The mechanisms are often ascribed to depletion of vesicle pools (Rizzoli and Betz, 2005), but their kinetic operating principles are not compatible with previous models and other explanations continue to be possible (Stevens and Wesseling, 1999a).

One of the mechanisms emerges after exhaustion of a readily releasable pool (RRP) of vesicles that can be released on demand. The other slows the overall rate at which vesicles are supplied to replenish the RRP but only begins to impact function after the release of more than two times the contents of the original RRP, which typically occurs only during extensive heavy drive. The slowdown was termed "supply-rate depression" (Garcia-Perez et

Received March 22, 2011; revised May 24, 2011; accepted June 15, 2011.

Author contributions: J.F.W. designed research; T.G., E.G.-P., K.M., J.G., R.M.-T., I.P.-0., and J.F.W. performed research; J.G., I.P.-0., D.C.L., and J.F.W. contributed unpublished reagents/analytic tools; T.G., R.M.-T., I.P.--0., and J.F.W. analyzed data; J.F.W. wrote the paper.

This work was supported by UTE project CIMA and grants from the Spanish Fondo de Investigaciones Sanitarias (PI060103) and Ministerio de Ciencia e Innovación (BFU2009-12160 and CSD2008-00005). We thank Aitor Zandueta Criado for expert technical assistance and Drs. Erwin Neher, Jorge Carneiro, Frantisek Supek, Robert Renden, Milos Petrovic, and Samuel Wang for helpful discussions and comments.

*T.G. and E. G.-P. contributed equally to this work.

Correspondence should be addressed to John F.Wesseling, Departamento de Neurociencias, Centro de Investigación Médica Aplicada, Universidad de Navarra, Pio XII, 55, 31008 Pamplona, Spain. E-mail: jwesseling@unav.es.

DOI:10.1523/JNEUROSCI.1447-11.2011

Copyright $\odot 2011$ the authors $\quad 0270-6474 / 11 / 3111563-15 \$ 15.00 / 0$ al., 2008). Alternative explanations that were considered but ruled out include $\mathrm{Ca}^{2+}$-channel inactivation, gradual vesicle priming, slow recruitment of $\mathrm{Ca}^{2+}$ channels to release sites, and action potential failures.

Supply-rate depression persists for minutes, causing slower RRP replenishment during subsequent rest intervals. However, replenishment always seems to proceed with the mathematical simplicity of the double exponential function $s(t)$ :

$$
s(t)=w \cdot\left(1-e^{\frac{-1}{\tau_{f}}}\right)+(1-w) \cdot\left(1-e^{\frac{-t}{\tau_{s}}}\right)
$$

where the time constants $\tau_{f}$ and $\tau_{s}$ have fixed values of $\sim 6.7 \mathrm{~s}$ and $\sim 1$ min, respectively. In contrast, the weighting parameter $w$ takes on values ranging from $\sim 1.0$ to $\sim 0.35$ that correspond to the amount of supply-rate depression at the beginning of the rest intervals after light to heavy use, respectively. Reports of more complicated recovery time courses are explained by additional factors operating independently of RRP replenishment, such as masked elements of short-term enhancement (Garcia-Perez and Wesseling, 2008).

The concept that RRP supply is driven by mass action of reserve vesicles is at the heart of previous models. However, the invariance of the time constants $\tau_{f}$ and $\tau_{s}$ in Equation 1 is not compatible with the mass-action concept (Stevens and Wesseling, 1999a), and instead suggests that the supply of vesicles to the RRP operates with bimodal kinetics. Almost all vesicles are ini- 
tially supplied by a fast mode characterized by $\tau_{f}$, whereas after the induction of supply-rate depression, some vesicles are supplied by an $\sim 10$-fold slower mode characterized by $\tau_{s}$. In contrast, mass-action mechanisms predict intermediate rates, which are not seen.

Pinpointing the function of individual presynaptic molecules will depend critically on the development of a comprehensive model of synaptic vesicle trafficking. Here, we begin by reporting new observations at wild-type (WT) synapses that extend and simplify the constraints that must be satisfied by any accurate model. We then propose a new theoretical model and a corresponding physical interpretation where supply-rate depression is ascribed to reserve pool depletion in a way that avoids massaction rules.

If the new model is accurate, manipulations that alter single rate-limiting mechanisms in vesicle trafficking should selectively alter one of the model's four parameters. Remarkably, we found that the synapsin 1 and 2 double knock-out (DKO) phenotype was fully explained by selectively altering the single parameter that determines the timing of induction of supplyrate depression.

\section{Materials and Methods \\ Animals}

Colonies of synapsin 1 and 2 knock-out mice were obtained from Jackson Labs and were crossed to obtain WT, DKO, and heterozygous strains (Rosahl et al., 1993, 1995). Both sexes were used in all experiments. Animals were killed by decapitation conducted in accord with Spanish Royal Decree 1201/2005.

\section{Electrophysiology}

All experiments were initiated after allowing synaptic transmission to rest for at least $4 \mathrm{~min}$, which was important because of the $\sim 1 \mathrm{~min}$ recovery time constant of supply-rate depression. Synaptic responses were measured from patch-clamped neurons held in whole-cell voltageclamp mode with pipette resistances of 3 to $5 \mathrm{M} \Omega$. Data were only accepted if the access resistance did not change detectably throughout individual experiments for slices and by $>10 \%$ for cell culture.

Slices. Experiments were performed on $400-\mu \mathrm{m}$-thick transverse hippocampal slices of 2- to 3-week-old mice, with the CA3 region removed, as described previously (Garcia-Perez and Wesseling, 2008). The extracellular recording solution contained $120 \mathrm{~mm} \mathrm{NaCl}, 1.25 \mathrm{~mm} \mathrm{NaH}_{2} \mathrm{PO}_{4}$, $26 \mathrm{~mm} \mathrm{NaHCO}_{3}, 10 \mathrm{~mm}$ glucose, $3.5 \mathrm{~mm} \mathrm{KCl}, 50 \mu \mathrm{m}$ picrotoxin, and 50 $\mu \mathrm{M}$ D-APV and was continuously bubbled with a mixture of $95 \% \mathrm{O}_{2}$ and $5 \% \mathrm{CO}_{2}$ starting at least 20 min before addition of $\mathrm{CaCl}_{2}$ and $\mathrm{MgCl}_{2}(2.6$ and $1.3 \mathrm{~mm}$ except where indicated). Recording pipettes were filled with solution containing (in mM) 130 Cs-gluconate, $5 \mathrm{CsCl}, 5 \mathrm{NaCl}, 2 \mathrm{MgCl}_{2}$, 2 MgATP, 0.2 LiGTP, 1 EGTA, $0.2 \mathrm{CaCl}_{2}$, and 10 HEPES, adjusted to a $\mathrm{pH}$ of 7.2 and an osmolarity of $290 \mathrm{mOsm}$. Synaptic responses were evoked with a monopolar silver/silver chloride stimulating electrode inserted into a glass pipette filled with recording solution (tip diameter, 10 to $30 \mu \mathrm{m}$ ) and placed in the Schaffer collaterals; stimulation intensity was a constant current of $\leq 0.1 \mathrm{~mA}$ for $\leq 0.1 \mathrm{~ms}$. Series resistance was not compensated, but measurement errors owing to imperfect space clamp were not a concern because of previous experiments showing that partial block of glutamate receptors with up to $1 \mathrm{~mm}$ kynurenic acid changes absolute but not relative sizes of responses during 20 or $40 \mathrm{~Hz}$ stimulation (Wesseling and Lo, 2002; Garcia-Perez et al., 2008). Each experiment in Figure 4 was run three times under standard conditions, and then three more times after application of $10 \mu \mathrm{M}$ DNQX to record stimulus artifacts in isolation. Experiments where access resistance changed between trials were discarded. The $\Delta T$ values in time course experiments (see Figs. 1C, 5, 6A, 9A) were shuffled. In Figure $8 A$, APV was removed, $10 \mu \mathrm{M}$ glycine was added, non-NMDA glutamate receptors were blocked with DNQX $(10 \mu \mathrm{M})$, and NMDA receptor currents (recorded at -10 $\mathrm{mV}$ ) were first monitored at low frequency ( $10 \mathrm{~s}$ intervals) to establish a baseline size, and again after $10 \mathrm{~min}$ in the presence of $40 \mu \mathrm{M}(+)-5$ -
methyl-10,11-dihydro-5H-dibenzo[a,d]cyclohepten-5,10-imine maleate (MK801).

Cell culture autapses. Autapses were cultured for 10 or $11 \mathrm{~d}$ from acutely dissected hippocampus from postnatal day 0 (P0) to P2 pups, and experiments were performed as described previously (Garcia-Perez and Wesseling, 2008), except the hypertonic solution used to induce osmotic shocks contained $2.6 \mathrm{~mm} \mathrm{Ca}^{2+}$ and $1.3 \mathrm{~mm} \mathrm{Mg}^{2+}$. Whole-cell recordings were via perforated patches achieved with $\sim 1 \mathrm{mg} / \mathrm{ml}$ amphotericin- $\mathrm{B}$ included in the pipette solution. The extracellular recording solution contained $132 \mathrm{~mm} \mathrm{NaCl}, 2 \mathrm{~mm} \mathrm{KCl}, 10 \mathrm{~mm}$ glucose, 15 mM sorbitol, $10 \mathrm{~mm}$ HEPES, $50 \mu \mathrm{M}$ D-APV, $2.6 \mathrm{~mm} \mathrm{CaCl}_{2}$ and $1.3 \mathrm{~mm}$ $\mathrm{MgCl}_{2}$. Recording pipettes were filled with the following (in mM): 140 K-gluconate, $9 \mathrm{NaCl}, 1 \mathrm{MgCl}_{2}, 2 \mathrm{MgATP}, 0.2 \mathrm{LiGTP}, 1$ EGTA, $0.2 \mathrm{CaCl}_{2}$, and 10 HEPES.

\section{Analysis of electrophysiology}

Response size was measured as the current integral. To calculate the total response over $100 \mathrm{~ms}$ or $1 \mathrm{~s}$ bins for Figure 4, the entire digitized traces with and without DNQX were first averaged across trials, and then the difference trace was calculated before integrating. RRP replenishment values in Figures 5 and $6 \mathrm{~A}$ were estimated as described previously (Garcia-Perez et al., 2008); the procedure requires that the second train of experimental pairs is sufficiently long to empty the RRP. Values in Figure $7 A$ were estimated as described previously (Stevens and Wesseling, 1998). Because preparations survived long enough for multiple measurements, $n$ values for Figures $1 C, 5,6$, and $9 A$ are greater than the number of preparations. However, to ensure accurate estimates, a detailed analysis showed no systematic trend, for any time point of any genotype, between recovery values measured at different times from the same preparation. Also, additional analyses where the first trial per preparation for each time point was considered alone yielded indistinguishable time courses, as did other analyses comparing recovery time courses to the time slices had spent in vitro before the experiment.

\section{Response size normalization}

Response sizes in Figures $2 B, 3$, and $8 B$ were normalized by the mean size of the first 20 responses because this allowed a comparison of the depression evident at later times. The more common method of normalizing by the size of the first response produced more scatter, owing to trial-to-trial fluctuations in the size of individual responses. In Figure $4 A$, response sizes were normalized by the mean size of responses 61 to 80 during $20 \mathrm{~Hz}$ stimulation to make the analysis directly comparable to Figure 1 of Garcia-Perez et al. (2008). In Figure 8 A, response sizes were normalized by the mean size of all 80 responses after MK801 application.

\section{FM1-43 imaging}

Primary neuronal cultures were grown from CA1 hippocampus dissected from P0 to P2 mouse pups and dissociated with papain (Worthington). Cells were plated on $15 \mathrm{~mm}$ coverslips (30,000 cells) that had been coated with poly-D-lysine and laminin. Cultures were grown in Neurobasal-A medium (Gibco) supplemented with 10\% horse serum, B27 (Gibco), 2 mм GlutaMax, and $1 \mu \mathrm{g} / \mathrm{ml}$ gentamycin. Experiments were performed 10 to $11 \mathrm{~d}$ after plating. The bathing solution contained the following (in mM): $130 \mathrm{NaCl}, 2 \mathrm{KCl}, 2.6 \mathrm{CaCl}_{2}, 1.3 \mathrm{MgCl}_{2}, 10$ glucose, 10 HEPES, 15 sorbitol, 1 kynurenate, and 0.05 picrotoxin, adjusted to $\mathrm{pH}$ 7.4. Staining and destaining were accomplished with field stimulation (20 Hz pulses of $10 \mathrm{~V}$ lasting $1 \mathrm{~ms}$ ) using a custom chamber designed as described previously (Burrone et al., 2006). Experiments were performed on an inverted microscope (IX71; Olympus) with a customized stage using a $40 \times$ air objective. Illumination was with a mercury lamp via an ND-25 (25\% transmission) filter and standard filter set (XF115-2; Omega Optical). Images were acquired at $1 \mathrm{~Hz}$ (100 ms exposure) with a CCD camera (Coolsnap HQ; Photometrix; $2 \times$ binning) using WinView32 software (Roper), and analyzed using ImageJ (NIH) and Matlab (Mathworks). The analysis in Figure 10 was accomplished by hand selecting the location of uniformly shaped 12 pixel $\left(1.3 \mu \mathrm{m}^{2}\right.$; quasicircular) regions of interest (ROIs) and after subtraction of background values obtained from each image. However, similar results were obtained when background subtraction values were instead estimated from the first image in each stack or when a computer algorithm was used to select 
A Sequence of
preliminary models

i.

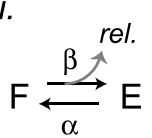

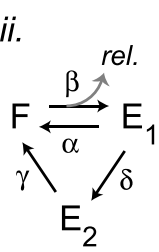

iii.
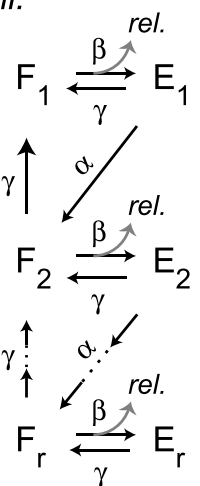

\section{B Working model}

$$
r=4 \text { (for WT) }
$$

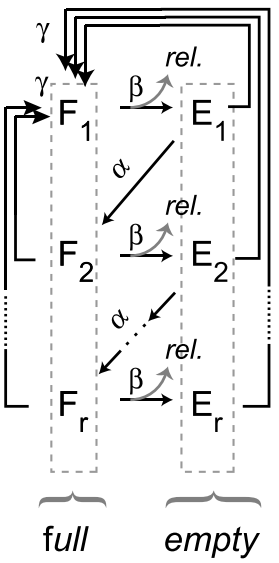

C
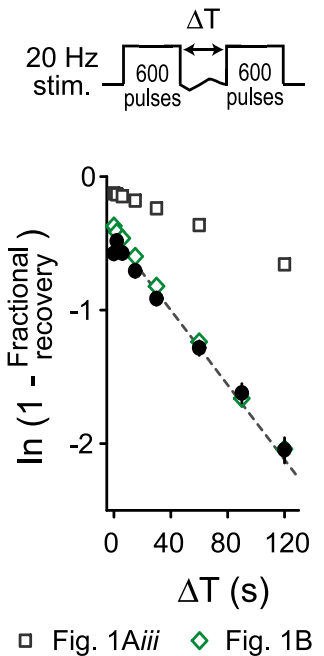

D
Physical interpretation of

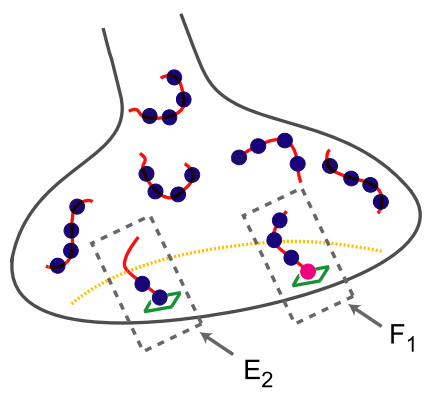

replenishment of reserves occurs by stochastic tether replacement

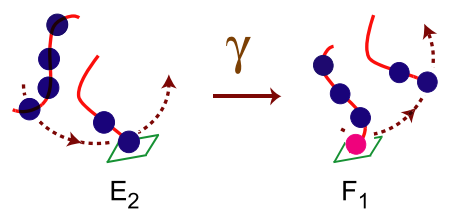

local reserve pool depletion of a single release site

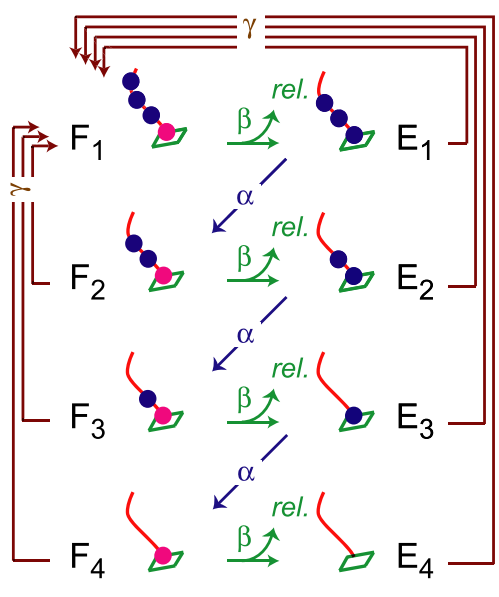

Figure 1. Development of a novel kinetic model for rate-limiting steps in vesicle trafficking. $A, B$, Markov models of single release sites. F states represent full states, meaning loaded with a docked and primed vesicle, whereas $E$ states represent empty states. rel. denotes release events, which occur during all transitions from $F$ to $E$ states. $A$, Key preliminary models that led to the working model. Ai, Two state Markov model depicting an individual release site. The model can account for key aspects of depression during the first several seconds of heavy drive, but does not account for the slowly recovering supply-rate depression induced later on. Aii, An extension with a secondary, more deeply inhibited $E$ state $\left(E_{2}\right)$. This model does predict a form of supply-rate depression, but cannot account for the delayed onset during induction seen in experiments. Aiii, A further extension that can account for the delayed induction of supply-rate depression in addition to the double exponential RRP replenishment time courses described in Equation 1, but not the additional observations in $\boldsymbol{C}$. $\boldsymbol{B}$, Working model. The parameter $r$ determines the length of the delay before onset of supply-rate depression by specifying the number of $F$ and Estate pairs. The total number of states would be $r^{*}$ 2. The difference compared to Aiiii is that the only transitions allowed from states with higher numbers to lower numbers are transitions into the $F_{1}$ state, which is key for satisfying the third constraint. C, The third constraint is $\tau=\tau_{s}$ recovery of the aggregate response during stimulation that induces the maximum amount of short-term depression. Maximal depression was induced at $S$ chaffer collateral synapses two times with a pair of $20 \mathrm{~Hz}$ stimulus trains of 600 pulses, separated by an experimentally varied rest interval $(\Delta T)$ as diagrammed at the top. Plotted is the logarithm of 1 minus recovery of the sum of all responses during the second train versus the intertrain interval $[n=24$ trials from 16 preparations, including the 12 used for Garcia-Perez et al (2008, their Fig. 5)]. The dashed line is the single exponential function with $\tau=70 \mathrm{~s}$, matching $\tau_{\mathrm{s}}$ in the previous study. Green diamonds are simulation results using the working model in $\boldsymbol{B}$ with $r=4$. Squares are the incorrect predictions made by the model in $\boldsymbol{A i i i i . ~} \boldsymbol{D}$, Schematic diagram of a reserve pool depletion interpretation of $\boldsymbol{B}$. Top left, Each release site (green squares) docks a vesicle-tethering unit (red strands). The theoretical model in $\boldsymbol{B}$ pertains to a single one of these sites (dashed gray boxes). Only one of the attached vesicles at a given site may be primed (magenta) at any given time, whereas the other vesicles function as a local reserve pool (blue). Individual release sites are modeled as behaving independently, and thus different sites can be in different states as depicted. Nondocked tethers are in the immobile reserve. Bottom left, the locations of ROIs. The destaining time courses of ROIs selected by the computer algorithm were slightly slower for both WT and $\mathrm{DKO}$, but other values were not different.

\section{Biochemistry}

Western blotting and quantification were conducted on tissue from 21-d-old female mice as described previously (Roberts et al., 2009), using a polyclonal anti-synapsin 1 and 2 antibody (Synaptic Systems).

\section{Simulations}

Simulations were performed with Matlab using the ode45 function for numerically integrating differential equations (integration interval, $50 \mathrm{~ms}$ ). The working model simulation for $r=2.6$ was calculated as the weighted sum of the simulation with $r=2$ (weighting, 0.4) and $r=3$ (weighting, 0.6).

\section{Results}

We began with the simplifying assumption that the RRP consists of a collection of independent release sites (Zampighi et al., 2008) and modeled the kinetic behavior of a single site. We searched for models that satisfied two important kinetic constraints: (1) the timing of RRP replenishment during rest intervals following the induction of depression is always well fit by Equation 1, and (2) the depression of the rate at which vesicles replenish the RRP (i.e., supply-rate depression) occurs with a delayed onset. Both of these constraints have been demonstrated experimentally but are not satisfied by previous models (Garcia-Perez et al., 2008).

We will not describe all models that were considered but ruled out when found to be incompatible with the two initial constraints. Instead, we begin by describing the logical sequence that finally led to our present working model.

\section{$\leftarrow$}

Replenishment of local reserve pools occurs when tethering units are spontaneously but infrequently replaced by new, fully loaded tethering units. Stochastic all-at-once replenishment such as this is a requirement of the result in C. Right, All possible states for a single release site/tethering unit complex in WT synapses during heavy drive, diagramed to match the scheme in $\boldsymbol{B}$. Arrows indicate the sequence of transitions between states. A release site with a primed vesicle is in one of the $\mathrm{F}$ states and releases the vesicle at the rate specified by $\beta$, transitioning the site on to the next $E$ state. Thereafter, a new vesicle will be primed from among the reserves attached to the tether at the rate specified by $\alpha$ if one is available. Stochastic replacement of tethering units is indicated here by the brown arrows pointing back to $F_{1}$ and occurs at the rate specified by $\gamma$. Priming (faster) and docking replacement tethers (slower) would be the two kinetic modes by which vesicles are supplied to the RRP. Instead of priming, $\alpha$ could alternatively be postfusion clearance of tangled SNAREs or physical translocation of vesicles along the tethers. 
Our previous working model [described previously by Wesseling and Lo (2002)] is presented in Figure $1 A i$ as a starting point. This model was designed to be compatible with key aspects of RRP supply during light and moderate synaptic drive and is similar to the widely used model of short-term plasticity of Tsodyks and Markram (1997), although expressed with a different formalism. According to the Markov formalism we use, release sites can be either in a full (F) state or an empty (E) state. Sites in the F state are loaded with a readily releasable vesicle, and transition to the E state occurs when the vesicle is spent via exocytosis. By definition, any given release site would be in one and only one of the states at any time (Meyn and Tweedie, 2009), implying that RRP size would equal the number of full sites. Sites in the $\mathrm{E}$ state then transition back to the F state when they are replenished with a new vesicle. The idea was that either docking or priming would be the rate-limiting step in supplying new vesicles for replenishment.

However, this model fails to provide a complete description of rate-limiting steps in vesicle trafficking because it cannot account for the supply-rate depression that is induced during extended drive. That is, all $\mathrm{E}$ state to $\mathrm{F}$ state transitions in Figure $1 A i$ are governed by the rate parameter $\alpha$, which required a value of $1 / \tau_{f}$ (i.e., $\sim 0.15 / \mathrm{s}$ ) to correctly predict the RRP replenishment time course after short or moderate amounts of drive. But a much slower rate parameter of $\sim 0.017 / \mathrm{s}$ would be required to explain the $\sim 1$ min value for $\tau_{s}$ in Equation 1. This was not a problem for modeling RRP replenishment after light or moderate drive because the $\tau_{s}$ component plays a negligible role, but becomes invalidating as soon as the $\tau_{s}$ component becomes significant, as it does after the induction of supply-rate depression ( $>6 \mathrm{~s}$ of stimulation at $20 \mathrm{~Hz}$ or faster).

We use the term "rate parameter" instead of "rate constant" because we considered models where activity-dependent modulation of rate parameters was allowed. However, although allowing $\alpha$ to decrease by a factor of three during extended drive could account for the supply-rate depression observed during stimulation, the slowly reversing changes in $\alpha$ that would be required to account for the corresponding slowdown in RRP replenishment during subsequent rest intervals were not compatible with the invariance of the time constants in Equation 1, which is the first constraint.

We next realized that adding a single deeply depressed state such as $\mathrm{E}_{2}$ in Figure 1 Aii could, in principle, account for both the supply-rate depression occurring during stimulation and the invariant time constants of Equation 1. In this case, the new rate parameter $\gamma$ would specify the transition from the $\mathrm{E}_{2}$ state back to the $\mathrm{F}$ state, and would have a value of $1 / \tau_{s}$ during rest intervals, whereas $\alpha$ during rest intervals would be $1 / \tau_{f}$. On average, sites in the $\mathrm{E}_{2}$ state would transition to the $\mathrm{F}$ state with the single exponential $y(t)=\left(1-e^{\frac{-t}{\tau}}\right)$ specified by $\tau=\tau_{s}$, whereas sites in the $\mathrm{E}_{1}$ state would transition to the $\mathrm{F}$ state with the analogous $\tau=$ $\tau_{f}$ single exponential. Because heavy stimulation would drive some release sites to $E_{1}$ and others to $E_{2}$, RRP replenishment as a whole would follow the sum of the two single exponentials, which is Equation 1. The parameter $w$ in Equation 1 would specify the fraction of sites in $\mathrm{E}_{1}$ at the beginning of the replenishment interval. Overall, RRP supply would be bimodal because no sites would transition to the $\mathrm{F}$ state with intermediate kinetics.

However, Figure 1 Aii predicts that the induction of supplyrate depression would follow a decaying exponential, analogous to radioactive decay, which conflicts with the second constraint requiring that induction occur with a delayed onset. We then realized that longer chains of repeated $\mathrm{F}$ and $\mathrm{E}$ states could be constructed in ways that would be consistent with the delayed onset of supply-rate depression, and the chains would maintain compatibility with the invariant time constants of Equation 1 if the $\mathrm{E}$ states were connected to $\mathrm{F}$ states by only two types of rateparameters (Fig. 1Aiii,B).

\section{Third constraint}

Next, to distinguish between multiple models that were consistent with the initial two constraints, we took advantage of the fact that different models differed dramatically in predicting the kinetic behavior of the deepest forms of short-term depression. For example, Figure 1 Aiii predicts that a deeper form would co-occur with supply-rate depression, causing supply-rate depression to be induced faster during a second episode of extensive synaptic drive than at completely rested synapses.

Experiments to test this were conducted in acute hippocampal slices and consisted of paired trains of $20 \mathrm{~Hz}$ Schaffer collateral stimulation, each lasting $30 \mathrm{~s}$ (600 pulses). The trains were separated in time by experimentally varied rest intervals as diagrammed at the top of Figure $1 C$, and neurotransmission was monitored with postsynaptic patch-clamp recordings of CA1 principle neurons, which provide linear measurements of neurotransmitter release (Garcia-Perez et al., 2008). Both trains induced the maximum amount of supply-rate depression.

For analysis, we quantified the recovery that occurred during each rest interval by dividing the aggregate response during the second train by the aggregate during the first train. This measure of recovery is different from measurements of RRP replenishment because the aggregate response is generated by the release of the entire reserve pool along with the RRP, which is approximately fourfold larger than the RRP alone (Harata et al., 2001; Garcia-Perez et al., 2008). Therefore, the resulting time course would strongly reflect the time it takes to replenish the reserve pool in addition to the much smaller RRP, which was already known to replenish according to Equation 1. In particular, models like Figure 1Aiii that predict forms of depression that are deeper than supply-rate depression would predict much slower recovery time courses than the models in Figure $1 B$.

Remarkably, the time course was approximated by a single exponential function:

$$
y(t)=(1-w) \cdot\left(1-e^{\frac{-t}{\tau}}\right)+w
$$

where $\tau=\tau_{s}$ from Equation 1 (Fig. $1 C$ ). The $\tau=\tau_{s}$ recovery of the aggregate response was therefore adopted as a third constraint and narrowed substantially the field of surviving models. Figure 1 Aiii was ruled out because it predicted a recovery time course that was an order of magnitude slower (Fig. 1C, squares). In fact, the only models known to us that are compatible with all three constraints constitute the class of closely related Markov chains diagrammed in Figure $1 B$ (see Fig. $1 C$, green diamonds underlying black data points).

\section{New kinetic framework for vesicle trafficking}

The Markov chains in Figure $1 B$ could be formalized with the following minimal set of differential equations:

$$
\frac{d F_{1}}{d t}=\gamma \cdot\left(\sum_{n=1}^{r} E_{n}+\sum_{n=2}^{r} F_{n}\right)-\beta \cdot F_{1},
$$




$$
\begin{gathered}
\frac{d F_{n}}{d t}=\alpha \cdot E_{n-1}-(\beta+\gamma) \cdot F_{n}, \text { for } n=2 \ldots r \\
\frac{d E_{n}}{d t}=\beta \cdot F_{n}-(\alpha+\gamma) \cdot E_{n}, \text { for } n=1 \ldots r-1, \\
\frac{d E_{r}}{d t}=\beta \cdot F_{r}-\gamma \cdot E_{r},
\end{gathered}
$$

where, as above, a release site in an F state would contribute a single vesicle to the RRP, and exocytosis of that vesicle would occur during the transition to the next $\mathrm{E}$ state. The rates of transition between states are specified by the rate parameters $\beta, \alpha$, and $\gamma$, which describe the speed of the rate-limiting steps in vesicle trafficking and release: $\beta$ specifies the transition rate from $\mathrm{F}$ to $\mathrm{E}$ states and thus the rate at which readily releasable vesicles undergo exocytosis. In contrast, $\alpha$ and $\gamma$ specify rates of transition back from $\mathrm{E}$ to $\mathrm{F}$ states, and therefore describe the two kinetic modes of RRP supply, and by extension the two rate-limiting steps in vesicle trafficking that emerge during heavy drive. The length of each Markov chain in the class is specified by a fourth parameter, $r$, which does so by specifying the number of $\mathrm{F}$ and $\mathrm{E}$ state pairs. Chains of any length are compatible with the first and third initial constraints, and all but the shortest chains predict a delayed onset for supply-rate depression, which is the second constraint. However, results presented below show that only the chain specified by $r=4$ could approximate the induction of supply-rate depression seen at WT synapses.

\section{Physical interpretation involving reserve vesicle pools}

The Markov chains in Figure $1 B$ are not compatible with previous vesicle-trafficking schemes, but are compatible with a reconceptualization of the reserve pool that additionally fits with tomography observations of what may be short vesicle-tethering units in presynaptic terminals (Siksou et al., 2007). This physical interpretation is presented in Figure $1 D$ to guide intuitions because Figure $1 B$ serves as the framework for our working model (below). The top left panel of Figure $1 D$ is a static depiction of a synaptic terminal with two release sites (green squares). Actual synapses have an average of $\sim 10$ (Schikorski and Stevens, 1997). Each site would dock a filamentous tethering unit, which could tether up to $r$ vesicles, where $r$ is the parameter that specifies the number of paired $\mathrm{F}$ and $\mathrm{E}$ states in the Markov chains. The diagram additionally shows nondocked tethering units loaded with vesicles, which have been seen in the tomography studies in addition to docked tethers (Siksou et al., 2007) and might constitute all or part of the immobile reserve pool of vesicles (Rizzoli and Betz, 2005).

The vesicle closest to the release site on docked tethering units would spontaneously prime for release (magenta), whereas the others (blue circles attached to docked filaments) would constitute a reserve pool dedicated exclusively for use at the release site. Heavy synaptic drive would eventually deplete the tether of vesicles because once the primed vesicle was released, the next reserve vesicle would take its place and become primed.

Reserve pools appear to replenish slowly when observed in bulk. However, the scheme in Figure $1 B$ requires that replenishment of tethers would occur all at once at individual release sites and never gradually or to partially full levels. In fact, models in Figure 1 Aiii failed to satisfy the third constraint because they predicted gradual replenishment, which would have been detected as a deep form of depression in Figure $1 C$ that was not seen. The slow reserve pool replenishment observed in bulk would thus arise from the low frequency at which individual tethers are replenished.

All-at-once reserve pool replenishment at individual release sites is accomplished in Figure $1 D$ (bottom left) by allowing partially or fully depleted docked tethering units to be spontaneously but infrequently replaced with completely loaded tethers. There would be no mechanism for only partially replenishing individual tethering units. Replacement tethers would be drawn either from the pool of nondocked tethers or from newly formed tethering units constructed from recycled vesicles. Importantly, however, for avoiding the mass-action concept, the rate of tether replacement would not decline during extensive synaptic use, implying that reconstitution of fully loaded tethers from recycled vesicles would be fast enough so that the supply of the replacements would never be limiting.

The kinetic framework in Figure $1 B$ pertains to a single one of the release sites attached to tethering units (Fig. $1 D$, top left, dashed boxes). Each release site would be in a single one of the states in Figure $1 B$ at any given time, but individual release sites in the same synaptic terminal could be in different states such as the two sites that are depicted.

The panel to the right shows all of the possible states for a single release site when $r=4$, connected together in a diagram matching Figure $1 B$. Release sites with fully loaded tethering units and a primed vesicle would be in state $F_{1}$. Action potentials would trigger the primed vesicle to release at the rate specified by $\beta$, which would temporarily transition the release site to $\mathrm{E}_{1}$ (green arrows). The site would then spontaneously transition on to $\mathrm{F}_{2}$ (blue arrows, $\alpha$ ), which would occur upon priming a new vesicle from the local reserve. Release and subsequent priming events with identical kinetics would then be repeated during continued synaptic drive until the tethering unit was completely exhausted of vesicles, which would correspond to $\mathrm{E}_{4}$. The infrequent, spontaneous replacement of tethering units (brown arrows, $\gamma$ ) could occur at any time from any state, and would transition the release site back to the $\mathrm{F}_{1}$ state.

\section{Accurate prediction of supply-rate depression}

We next tested whether the kinetic framework in Figure $1 \mathrm{~B}$ could accurately predict short-term depression during heavy synaptic drive. The values of $\beta, \alpha$, and $\gamma$ that have already been constrained by previous results are summarized in Figure $2 A$. The origin of each estimate is referenced in the figure legend. Briefly, $\beta$ must be close to zero during rest intervals to account for the low spontaneous release rate, and much faster than $\alpha$ or $\gamma$ during 20 $\mathrm{Hz}$ stimulation because this is a condition for RRP exhaustion, which has been verified experimentally (Garcia-Perez et al., 2008). Parameters $\alpha$ and $\gamma$ correspond to $\tau_{f}$ and $\tau_{s}$ in Equation 1 during rest intervals; specifically, $\alpha=\frac{1}{\tau_{f}}-\gamma$ and $\gamma=\frac{1}{\tau_{s}}$ (derivation not shown). In addition, $\alpha$ would be moderately faster during stimulation because of activity/residual $\mathrm{Ca}^{2+}$. dependent acceleration that is known to occur (Stevens and Wesseling, 1998); this is compatible with the invariance of the time constants in Equation 1 (see below). However, whether or not $\gamma$ is likewise modulated by activity was not explicitly constrained by previous results.

The value of $r$ was not explicitly constrained by previous results either. However, only the chain of $r=4$ pairs of $\mathrm{F}$ and $\mathrm{E}$ states could approximate the induction of supply-rate depression observed during heavy drive at WT synapses (Fig. 2 B). Even so, the prediction did not perfectly match the observed steady-state response size when $\gamma$ was assumed to be insensitive to modula- 
Constraints on parameter values

\begin{tabular}{c|c|c} 
& active & resting \\
\hline$\beta$ & $\begin{array}{c}\text { fast } \\
(>5 / \mathrm{s})\end{array}$ & 0 \\
\hline$\alpha$ & $0.22 / \mathrm{s}^{*}$ & $\begin{array}{c}0.13 / \mathrm{s}^{*} \\
\left(\tau_{f} \sim 6.7 \mathrm{~s}\right)\end{array}$ \\
\hline$\gamma$ & $?^{* *}$ & $\begin{array}{c}0.017 / \mathrm{s} \\
\left(\tau_{s} \sim 1 \mathrm{~min} .\right)\end{array}$
\end{tabular}

$\beta$ during first 4 s of $20 \mathrm{~Hz}$ stim.

B
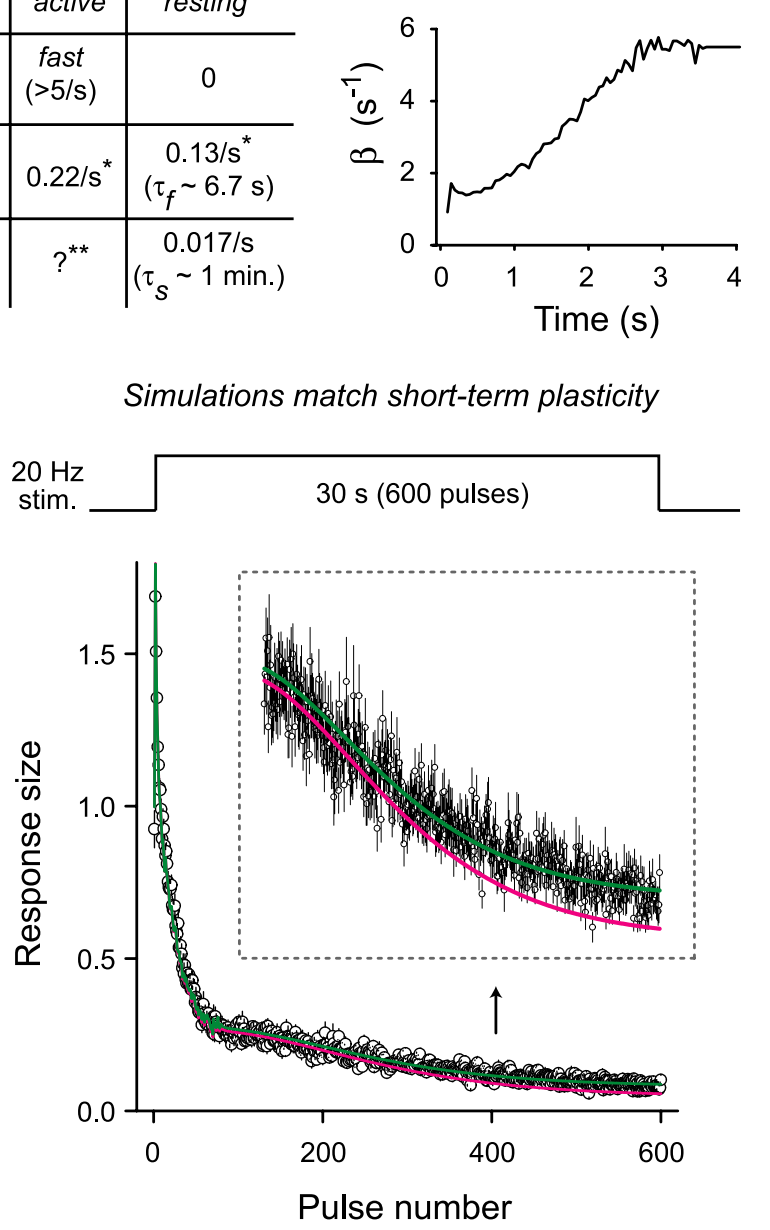

Figure 2. The working model accurately predicts the induction of supply-rate depression. $A$, Left, Values for rate parameters in the working model that were constrained by previous experiments. Increasing the resting value for $\beta$ to match the estimates of the spontaneous minirate $(<0.0005 / \mathrm{s}$ per release site) made no detectable difference in the results. Single asterisks indicate that values for $\alpha$ in Wesseling and Lo (2002) correspond to $\alpha+\gamma$ here. The resting value for $\gamma$ was calculated assuming $\tau_{s}=1 \mathrm{~min}$, which is close to both the value in cell culture using pairs of osmotic shocks (Stevens and Wesseling, 1999a) and the value newly estimated from Figure 5 in the present report, but slightly faster than the $\tau_{\mathrm{s}}=70 \mathrm{~s}$ value reported by Garcia-Perez et al. (2008). Double asterisks indicate that $\gamma$ needed to be accelerated to 0.025 /s during active periods for the working model to match observations exactly; this value was not included in the table because it was not highly constrained by data in previous reports, and is therefore a prediction of the working model, rather than a constraint. Right, Values for $\beta(t)$ used during modeling throughout the present report. Values were calculated from the first 80 responses plotted in $\boldsymbol{B}$ using the kinetic scheme described in the appendix of the study by Wesseling and Lo (2002), assuming only that the RRP is depleted to a standing fullness of $\sim 5 \%$ by the end of the first $3 \mathrm{~s}$ of $20 \mathrm{~Hz}$ stimulation. $\boldsymbol{B}$, Short-term plasticity during $30 \mathrm{~s}$ of $20 \mathrm{~Hz}$ Schaffer collateral stimulation in slices ( $n=66$ preparations). Response sizes were normalized by the mean size of the first 20 responses. The magenta line is the simulation of the working model with $r=4, \gamma=0.017 / \mathrm{s}$, and the other parameters with the values specified in $\boldsymbol{A}$. The green line is the same model with $\gamma$ accelerated to $0.025 / \mathrm{s}$.

tion by activity (Fig. $2 B$, magenta lines). Instead, a precise fit was obtained by increasing $\gamma$ from the resting value of $1 / \tau_{s}(\sim 0.017 / \mathrm{s})$ to $0.025 / \mathrm{s}$ (Fig. $2 B$, green lines), which suggests the presence of modest activity-dependent acceleration that might be analogous to the acceleration of $\alpha$ that has already been characterized. Based on the close fits to observations and the lack of known alternatives, we therefore adopted as our working model the model in Figure $1 B$, where $r=4 ; \beta, \alpha$, and $\gamma$ have the values listed in Figure $2 A$; and additionally, $\gamma$ is accelerated to $0.025 / \mathrm{s}$ by activity.

For simplicity we modeled acceleration and deceleration of both $\alpha$ and $\gamma$ as occurring instantaneously at the beginning and end of stimulation even though results from osmotic shock experiments in cultured autapses suggest that the mechanism underlying the acceleration of $\alpha$ may actually operate more gradually (Stevens and Wesseling, 1998) owing to the gradual accumulation and clearance of residual $\mathrm{Ca}^{2+}$ (Garcia-Perez and Wesseling, 2008). The simplification works, however, because (1) $\alpha$ quickly reaches a maximal value in $<1$ s during continuous 20 $\mathrm{Hz}$ (or faster) stimulation and can be treated as a constant while stimulation persists to within a second-order correction for modeling short-term depression (Wesseling and Lo, 2002), and (2) the expected deviation of the faster component from a single exponential during RRP replenishment is small, and importantly, the size of the deviation remains comparable across the entire range of experimental conditions, including when $w$ in Equation 1 has a low value and the $\tau_{s}$ component plays a dominant role (see Fig. 5Bii) (see also Garcia-Perez et al., 2008, their Fig. 6C). A more precise formulation for the faster component of RRP replenishment that accounts for the deviation would be $\left(1-e^{-\int \alpha(t) d t}\right)$ rather than $\left(1-e^{\frac{-t}{\tau_{f}}}\right)$ in Equation 1 (Wesseling and Lo, 2002, their Equation 2) (see Fig. 5Bii, solid green line), but this is also a second-order correction with little impact on the present findings.

\section{Compatibility with manipulations that alter release rates}

The working model is not intended to supplant current concepts about how transmitter release is triggered, but instead remains neutral (Lou et al., 2005; Moulder and Mennerick, 2005; Schneggenburger and Neher, 2005; Sun et al., 2007; Pan and Zucker, 2009). For simplicity, the model does treat the release of readily releasable vesicles as a single trafficking step that accelerates from 0 during rest periods to fast enough to exhaust the RRP during periods of heavy drive. However, the value of the model for identifying upstream rate-limiting steps in vesicle trafficking depends critically only on the already verified premise that repetitive stimulation can drive release fast enough to maintain the RRP in a near-empty steady state, regardless of the kinetic complexity of the release trigger (Garcia-Perez et al., 2008).

\section{Selective permutations of single parameter values}

Our next goal was to test the working model further. We reasoned that if the three rate parameters and $r$ each pertain to a genuine rate-limiting element in vesicle trafficking, then some experimental manipulations known to affect short-term synaptic plasticity might alter selectively the value of one of these parameters without affecting the values of the other three, and without requiring additional complexity. Indeed, increasing the stimulating frequency or extracellular $\mathrm{Ca}^{2+}$ increased $\beta$ values selectively (data not shown) as expected, because these manipulations increase the rate at which vesicles within the RRP are released but do not affect the size of the RRP, the baseline rate of supply, or the induction of supply-rate depression (Garcia-Perez et al., 2008). Moreover, although no selective manipulations of $\alpha$ or $\gamma$ are yet known, the fourth parameter, $r$, is shown next to be selectively altered in synapsin knock-outs. 
A

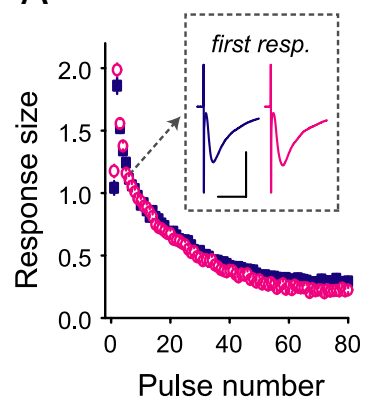

B

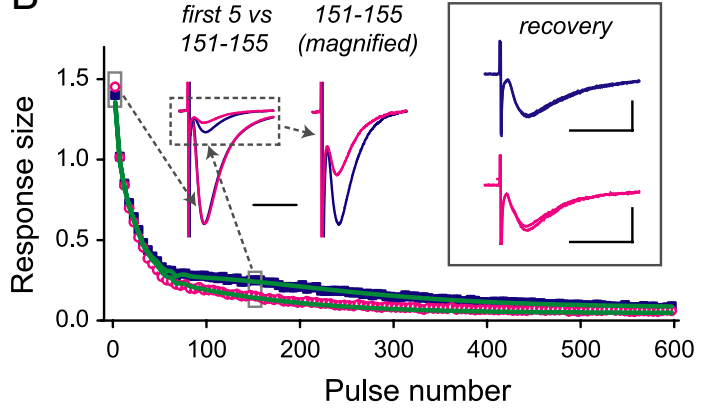

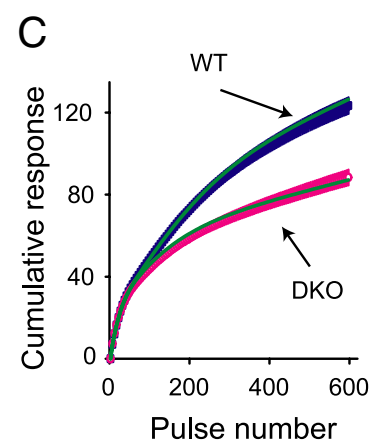

Figure 3. Synapsin DKO synapses depress faster and more extensively during heavy stimulation. Responses during $30 \mathrm{~s}$ of $20 \mathrm{~Hz}$ Schaffer collateral stimulation in slices from strain-matched DKO and WT animals $(n=55$ preparations for DKO; WT values are from Fig. 2B). Magenta symbols represent DKO, and blue represents WT. A, First 80 responses. Insets are the first responses averaged across all experiments. Calibration: 100 pA, 25 ms. B, All 600 responses versus pulse number, binned into groups of five for clarity. Error bars (SEM) are smaller than the symbols. Green lines are the working model with $r=2.6$ (matching DK0) and $r=4$ (WT). Left inset, Averaged traces across all experiments of responses 1 to 5 and 151 to 155 , scaled by the mean size of responses 1 to 5 . Scale bar, $25 \mathrm{~ms}$. Middle inset, Magnified responses 151 to 155 . Boxed inset, Overlaid, averaged traces from an additional set of experiments before and at least $4 \mathrm{~min}$ after $600 \mathrm{pulses}$ ( $20 \mathrm{~Hz}$; average of 5 experiments). Calibration: 100 pA, 20 ms. C, Cumulative responses. Green lines are the working model.

\section{Confirmation of more short-term depression in synapsin knock-outs}

We chose to analyze synapsin knock-outs against the working model because of previous reports implicating synapsins in vesicle trafficking and short-term depression (Huttner et al., 1983; Pieribone et al., 1995; Gitler et al., 2008). We conducted the analysis primarily in slices instead of cell cultures because the absence of asynchronous release at Schaffer collateral synapses in slices allows for more precise kinetic measurements of short-term depression in release rates (Garcia-Perez et al., 2008). However, some experiments that required cell cultures are included below.

Figure 3 confirms previous reports (Rosahl et al., 1995; Jensen et al., 2007) that DKO synapses exhibit more depression than WT synapses during extensive repetitive stimulation. Schaffer collaterals were stimulated for 600 pulses at $20 \mathrm{~Hz}$ because this protocol induces the maximum amount of depression and because $20 \mathrm{~Hz}$ stimulation maintains the RRP in a near-empty steady state at WT synapses, which allows rate-limiting mechanisms to be isolated from other factors contributing to depression (Garcia-Perez et al., 2008).

The amount of depression evident in the responses to the first 40 pulses did not differ between DKO and WT synapses (Fig. $3 A, B$ ), but a difference began to emerge after the 60th pulse and was largest after about 150 pulses (Fig. 3B), with DKO synapses depressing to $15 \pm 1.0 \%$ of their initial strength, versus $25 \pm$ $1.1 \%$ for WT (mean \pm SEM; $p<1 E-8$ ). The difference subsequently narrowed with DKO synapses depressing to steady-state levels $(5.8 \pm 0.7 \%)$ that were again lower than WT synapses $(9.8 \pm 1.0 \% ; p<0.002)$. Overall, the additional depression yielded cumulative responses over the entire 600-pulse trains that were $\sim 35 \%$ lower at DKO synapses (Fig. $3 C$ ). Over several minutes of rest, both DKO and WT synapses recovered completely (Fig. $3 B$, inset), confirming that the defect was in some aspect of short-term plasticity.

The additional depression at DKO synapses would be expected to reflect a presynaptic alteration because synapsins are primarily located in presynaptic terminals and because shortterm depression seems to be completely presynaptic at WT Schaffer collateral synapses (Wesseling and Lo, 2002; Garcia-Perez et al., 2008). In fact, decreases were detected in the probability of release but not quantal size during near-minimal stimulation experiments (data not shown).

The reduction in the aggregate response seems to match with the similarly reduced vesicle count in DKO terminals (Rosahl et al., 1995) but might have been caused by an unrelated mechanism because only a minority of the vesicles identified morphologically participate in neurotransmission (Harata et al., 2001). Indeed, other mechanisms have been proposed (Sun et al., 2006). Nevertheless, an FM1-43 imaging experiment presented below (see Reserve pool depletion mechanism for supply-rate depression) confirms that DKO synaptic terminals do exhibit reduced recycling reserve pool sizes.

\section{Evaluation of three potential causes for the additional depression at DKO synapses}

In principle, the additional depression could have been caused by any of the following: (1) the absence of a subdivision of the RRP that is ordinarily released only during extensive stimulation (i.e., during stimulation that continues after the first 60 pulses), similar to a previously suggested explanation at calyx of Held synapses (Sun et al., 2006); (2) use-dependent decreases or fatigue in the efficiency of the release machinery such that some readily releasable vesicles that would ordinarily be released at WT synapses would be left unreleased [in principle, such a decrease could be caused by any of several mechanisms that do not limit release at WT synapses when stimulation is $20 \mathrm{~Hz}$ or faster but might at DKO synapses (Garcia-Perez et al., 2008), such as $\mathrm{Ca}^{2+}$-channel inactivation (Hennig et al., 2008; Mochida et al., 2008), gradual vesicle priming (Wu and Borst, 1999), or slow recruitment of $\mathrm{Ca}^{2+}$ channels to release sites (Wadel et al., 2007)]; or (3) slower RRP resupply, leading to a decrease in the maximum amount of release over extended periods, which might be consistent with a previously proposed synapsin hypothesis (Greengard et al., 1993).

Possibility (1) seemed unlikely because previous experiments showed that the first 60 pulses exhaust the entire RRP at WT synapses and that subsequent release comes from vesicles that are newly supplied to the RRP (Garcia-Perez et al., 2008). We therefore focused initially on distinguishing between possibilities (2) and (3).

We reasoned that if the additional depression seen at DKO synapses was caused by decreased efficiency of the release machinery, which is possibility (2), then the unreleased vesicles would accumulate within the RRP. The amount of accumulation would be substantial because the $\sim 35 \%$ smaller cumulative response seen at DKO synapses during $30 \mathrm{~s}$ of stimulation (Fig. 3C) would correspond to a difference of approximately two times the contents of the RRP when full; that is, over the same interval, WT 


\section{A 20 vs $40 \mathrm{~Hz}$ continuous stimulation}

i.

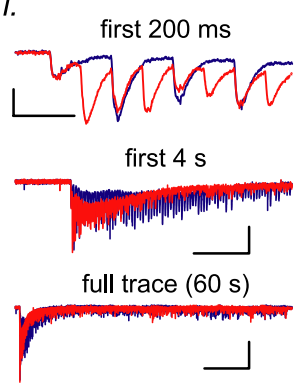

ii.

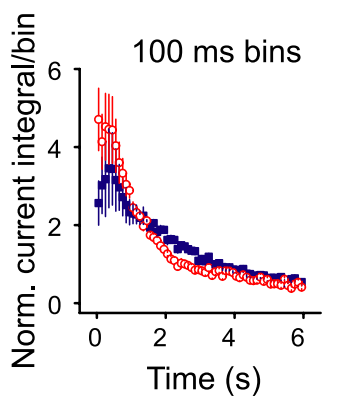

iii.

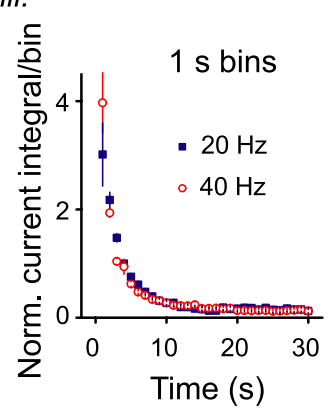

B Frequency jumps

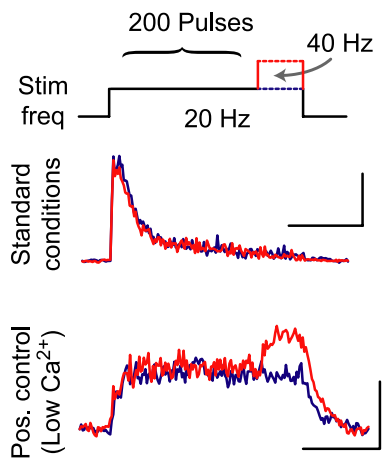

Figure 4. Sixty-pulse trains are long enough to exhaust the RRP at DK0 synapses. $A$, Schaffer collaterals were driven at 20 or $40 \mathrm{~Hz}$ for $60 \mathrm{~s}$ ( 1200 or 2400 pulses; 8 paired trials from 5 preparations). Ai, Traces from an example experiment after subtracting the stimulus artifacts (see Materials and Methods). Top, First $200 \mathrm{~ms}$. Calibration: 100 pA, 50 ms. Middle, First 4 s. Calibration: 100 pA, $1 \mathrm{~s}$. Bottom, All 60 s. Calibration: $100 \mathrm{pA}, 10 \mathrm{~s}$. Aii, Mean of the current integral per $100 \mathrm{~ms}$ bin during the first $6 \mathrm{~s}$ of stimulation across all experiments (bin values were normalized by the mean size during the fourth $s$ of stimulation at $20 \mathrm{~Hz})$. Note that the time-integrated response was greater at $40 \mathrm{~Hz}$ during the first $1 \mathrm{~s}$ of stimulation $(140 \pm 9 \%)$, which confirms that stimulation at $40 \mathrm{~Hz}$ can release neurotransmitter at a faster rate before the RRP has been exhausted. The difference disappeared during the first $3 \mathrm{~s}$ of stimulation (106 $\pm 5 \%$ at $40 \mathrm{~Hz}$ ). Aiii, Mean of response integral per $1 \mathrm{~s}$ bin over the first $30 \mathrm{~s}$ of stimulation showing that after the fourth second the response integral matched at the two stimulation frequencies, even in the face of a more than threefold further decline. The individual responses at $40 \mathrm{~Hz}$ were half the size of the responses at $20 \mathrm{~Hz}$, but there were twice as many. Overall, no difference was detected in the cumulative response integral over $30 \mathrm{~s}$ of stimulation $(101 \pm 8 \%)$. See Figure 1 of Garcia-Perez et al. (2008) for equivalent experiments at WT synapses. B, Stimulation frequency was doubled after the 200 th pulse at $20 \mathrm{~Hz}$. No increase in the time-integrated response was detected under standard conditions (top; $n=4)$, but a robust increase was detected in positive controls conducted at a reduced $\mathrm{Ca}^{2+} / \mathrm{Mg}^{2+}$ ratio $(0.45 / 2.5 \mathrm{~mm}$ ), where $20 \mathrm{~Hz}$ stimulation does not exhaust the RRP (bottom; $n=8$ ). The response integral during the first $2 \mathrm{~s}$ following the frequency jump compared to first $2 \mathrm{~s}$ overall was $129 \pm 18 \%$, compared to $87 \pm 9.5 \%$ with no jump $(p<0.01)$. Traces are from example experiments. Calibration: top, $30 \mathrm{pA}, 5 \mathrm{~s}$; bottom, $10 \mathrm{pA}, 5 \mathrm{~s}$.

synapses released the equivalent contents of $5.5 \pm 0.2 \mathrm{RRPs}$, which was calculated from the data in Figure 3 using the theory described by Wesseling and Lo (2002, their appendix) to estimate the cumulative response to the release of the equivalent contents of a single RRP. Therefore, more intense stimulation that was more effective at releasing the unreleased vesicles would be expected to yield a larger response.

To test this, we drove DKO synapses with higher-frequency stimulation. No increase in the cumulative response was detected when stimulation was 40 instead of $20 \mathrm{~Hz}$ over trials of continuous stimulation lasting $60 \mathrm{~s}$ (Fig. $4 \mathrm{~A}$ ) or when the stimulating frequency was abruptly increased from 20 to $40 \mathrm{~Hz}$ after the first 200 pulses (Fig. $4 B$ ), which is when the difference between DKO and WT is large. However, increasing the stimulating frequency did produce robust increases in responses in positive control experiments where RRP exhaustion was prevented by reducing extracellular $\mathrm{Ca}^{2+}$ (Fig. 4B), confirming basic features of the experimental paradigm such as the capacity of axons to fire at higher frequencies.

These results argue against the presence of a substantial unreleased population of releasable vesicles, and thus do not support possibility (2). In contrast, the matching cumulative response observed during 20 and $40 \mathrm{~Hz}$ stimulation would be predicted if stimulation at either frequency were enough to drive release maximally by first exhausting the RRP during the first few seconds of stimulation as occurs at WT synapses. Therefore, the results are consistent with possibility (3) that the additional depression at DKO synapses is caused by the emergence of one or more slow vesicle-trafficking steps governing RRP resupply, possibly caused by earlier depletion of a smaller reserve pool. The remaining caveat would be that the hypothetical fatigue in the release machinery in possibility (2) was extreme enough to prevent completely the release of a large fraction of readily releasable vesicles, but this caveat would not explain the observation of slower RRP replenishment after intermediate amounts of stimulation that is presented next.

\section{Slower RRP replenishment after moderate-length trains}

Possibility (3) predicts that RRP replenishment would take longer at DKO compared to WT synapses. No such difference has been detected (Sun et al., 2006), but replenishment time courses in previous studies were measured after releasing the equivalent contents of a single RRP and might have missed defects in replenishment that emerge during more extended drive. In fact, supplyrate depression is not detectable at WT synapses until after the equivalent contents of more than two RRPs have been released (Garcia-Perez et al., 2008). It was therefore possible that the additional depression at DKO synapses was caused by the earlier induction of supply-rate depression, although not early enough for detection after light stimulation.

We found this to be the case in a series of additional experiments in slices. For example, 80 -pulse stimulus trains released the equivalent contents of $1.9 \pm 0.04$ RRPs (slightly less at DKO synapses), and subsequent RRP replenishment was slower at DKO compared to WT synapses (Fig. 5A). A higher-resolution analysis revealed that the DKO replenishment time course was well fitted by Equation 1, although with a lower value for the parameter $w$ than the fit for WT data. Equation 1 additionally fit the much slower replenishment time courses that followed trains of 600 pulses. A detailed analysis indicated that whereas differences between replenishment time courses at DKO and WT synapses can be ascribed to differences in the value of the weighting parameter $w$, they cannot be ascribed to changes in either of the component time constants (Fig. 5B).

\section{Earlier onset of supply-rate depression}

Since the value of $w$ is proportional to the amount of supply-rate depression induced during the preceding stimulus train (GarciaPerez et al., 2008), the results so far suggest that the difference between DKO and WT might be limited to the timing of induction of supply-rate depression. Therefore, we next measured the value of $w$ as a function of the length of the preceding stimulus train. A decrease in $w$ was detected after trains as short as 60 


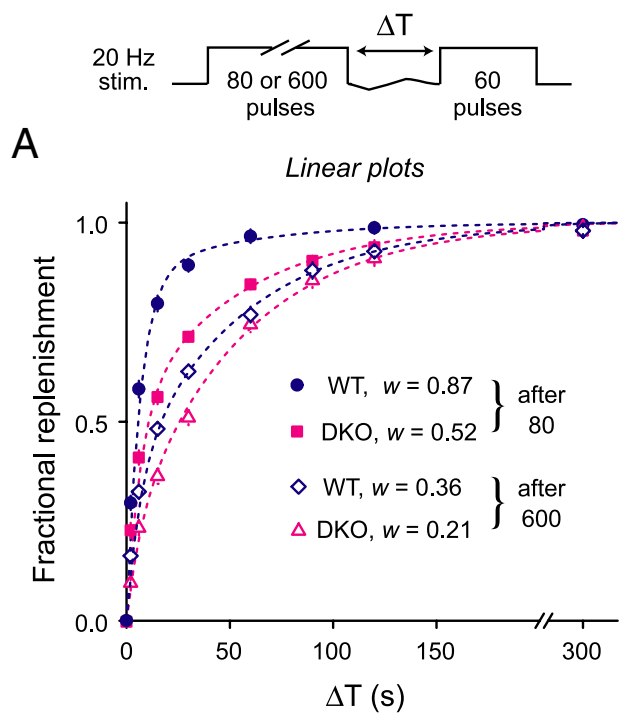

B
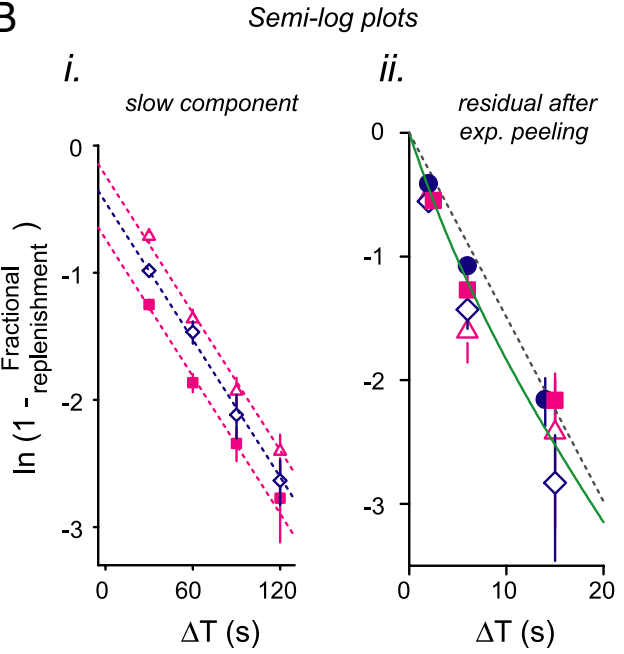

Figure 5. Slower RRP replenishment after moderate drive, but unaltered recovery kinetics of component exponentials. RRP replenishment time courses were measured after 80 or 600 pulses with pairs of $2 \mathrm{OHz}$ stimulation trains separated in time by experimentally varied intervals as diagrammed at the top (WT after 80 pulses, $n=45$ trials from 23 preparations; WT after 600 , $n=24$ trials from 16 preparations; DK0 after $80, n=47$ trials from 34 preparations; DKO after $600, n=42$ trials from 31 preparations). $\boldsymbol{A}$, Linear plots showing that RRP replenishment is slower after 80 pulses at DKO compared to WT synapses. Dashed lines are Equation 1 with $\tau_{f}=6.7 \mathrm{~s}, \tau_{\mathrm{s}}=55 \mathrm{~s}$, and $w$ as indicated. $\boldsymbol{B}$, Semilogarithmic plots. $\boldsymbol{B i}$, Later time points gathered for $\Delta T$ values between 30 and $120 \mathrm{~s}$. Dashed lines are the single exponential $y(t)=(1-w) \cdot\left(1-e^{\frac{-t}{\tau}}\right)+w$, with $\tau=55 \mathrm{~s}$ and $w$ values as in $A$. Best-fitting time constants forjust the later data points were calculated as $55 \mathrm{~s}$ (range of $46 \mathrm{t}$ to $69 \mathrm{~s}$ with $95 \%$ confidence) for WT after 600 pulses, $56 \mathrm{~s}$ ( 43 to $77 \mathrm{~s}$ ) for DK0 after 600 pulses, and $54 \mathrm{~s}$ ( 45 to $65 \mathrm{~s})$ for DKO after 80 pulses. Bii, Earlier time points after first subtracting the slower exponential component assuming $\tau=55 \mathrm{~s}$. The dashed gray line is the single exponential with $\tau=6.7 \mathrm{~s}$. Deviations from the single exponential were expected because of the activity/residual $\mathrm{Ca}^{2+}$. dependent acceleration of $\alpha$, which reverses as the $\mathrm{Ca}^{2+}$ is cleared during rest intervals. The solid green line is the deviation predicted by Wesseling and Lo (2002, their Eq. 2), with $N=1$ and assuming $\alpha$ is accelerated from $0.10 / \mathrm{s}$ to $0.24 / \mathrm{s}$ by residual $\mathrm{Ca}^{2+}$ and that subsequent clearance during rest intervals follows the time course in Figure 2D of Garcia-Perez and Wesseling (2008). The WT data point after 80 pulses for $\Delta T=15 \mathrm{~s}$ and the DKO data point after 80 pulses for $\Delta T=2$ s are shifted slightly along the horizontal axis to make them visible.

pulses at DKO synapses (Fig. 6A), whereas no significant decrease was detected at WT synapses until after the 200-pulse trains. The results are plotted against the cumulative amount of release during the preceding stimulus train because supply-rate depression is thought to depend on the amount of release rather than the amount of stimulation per se (Stevens and Wesseling, 1999a). Further analysis confirmed that values for $w$ were proportional to the later phase of depression arising during stimulation for DKO synapses (data not shown), matching WT behavior. Together, these results indicate that the DKO phenotype evident in Figures 3,5 , and $6 A$ is likely caused by an earlier onset of supply-rate depression, further supporting possibility (3) above.

\section{Synapsin 2 dosage dependence of induction}

We next devised a single-point assay for comparing the amount of supply-rate depression induced by trains of 120 pulses $(20 \mathrm{~Hz})$ in a range of genotypes. Trains of 120 pulses were chosen because they produced the largest difference between DKO and WT (Fig. $6 A)$. The idea was that more supply-rate depression in the assay would indicate earlier induction.

The severity of the phenotype was found to depend strongly on the dosage of synapsin 2, but not synapsin 1 (Fig. 6 Bi). Quantitative Western blot analysis of hippocampal extracts confirmed that synapsin 2 protein expression is reduced by $\sim 50 \%$ in synap$\sin 2$ heterozygotes (Fig. 6 Bii), matching the $\sim 50 \%$ magnitude of the effect on supply-rate depression. Further experiments confirmed that RRP replenishment time courses continued to be well fitted by Equation 1 at synapsin 2 knock-out synapses (data not shown). The lack of an effect of knocking out synapsin 1 fits with previous studies where exogenous synapsin $2 \mathrm{a}$ expression rescued a defect in short-term depression in synapsin 1, 2, and 3 triple knock-outs, whereas synapsin 1 expression did not (Gitler et al., 2008).

\section{Cell culture confirmation of slower RRP replenishment after moderate use}

Although our overall goal was to test the working model against the synapsin knock-out phenotype, the intermediate conclusion that synapsins are involved in vesicle trafficking is important on its own because of the present uncertainty regarding whether synapsins instead primarily control vesicle release probability by a downstream mechanism (Greengard et al., 1993; Sun et al., 2006). Because the previous evidence against a vesicle-trafficking phenotype was the lack of alterations in the time course of RRP replenishment, we sought to confirm slower replenishment using osmotic shocks instead of electrical stimulation to measure replenishment (Stevens and Wesseling, 1999a). The experiment had to be conducted in autapses grown in cell culture instead of slices because autapses allow the simultaneous electrical activation of all afferent synapses, and osmotic shocks can be conveniently induced with hyperosmotic solution challenges (Fig. 7A, top).

Less replenishment was observed at DKO compared to WT synapses over the 20-s-long rest intervals that followed trains of 80 action potentials $(0.78 \pm 0.11$ vs $0.96 \pm 0.10 ; n=6$ trials/ autapses each; $p<0.02$ ) (Fig. 7A, dashed box). Conceptually, the 80 -action-potential experiment approximates the single-point assay indicated in Figure $6 \mathrm{~A}$ and yielded a similar difference between DKO and WT. Further experiments confirmed that the full RRP replenishment time course continued to be fit by Equation 1 when measured with osmotic shocks (Fig. 7A, bottom).

These experiments provide independent confirmation that the DKO phenotype includes an alteration in vesicle trafficking upstream of release because osmotic shocks trigger release by an artificial mechanism that bypasses the $\mathrm{Ca}^{2+}$-dependent trigger of the native release machinery (Rosenmund and Stevens, 1996). We emphasize, however, that our results are consistent with pre- 
A
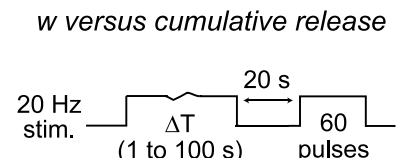

(20 to 2000 pulses)

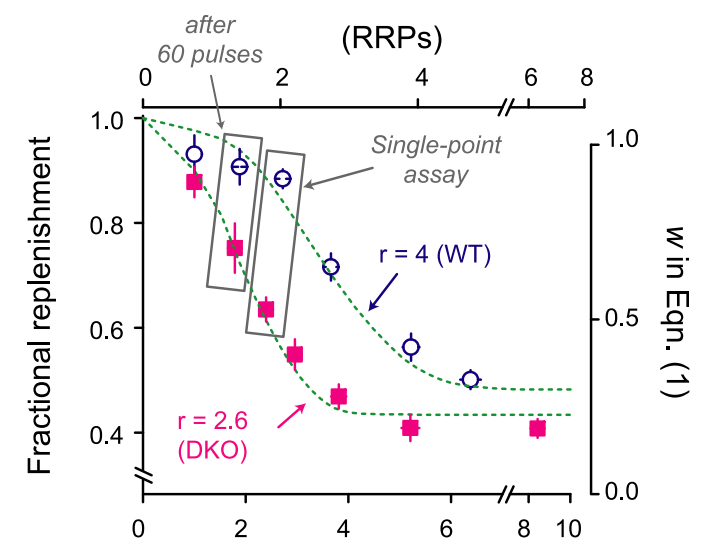

Cumulative release during $1^{\text {st }}$ train

(Relative to $1^{\text {st }} 20$ resps.)

B

\section{Single-point assay of dosage}

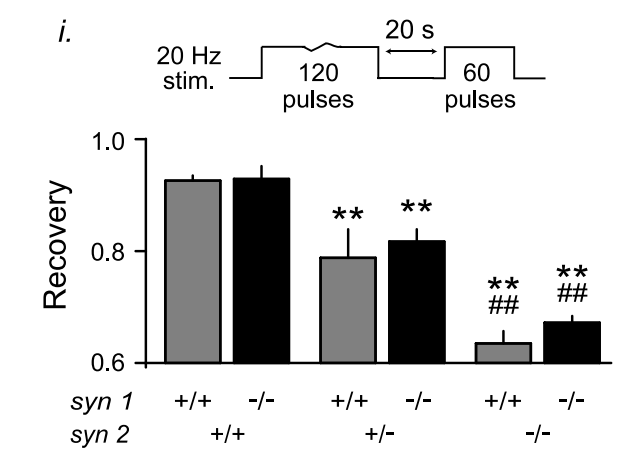

ii.

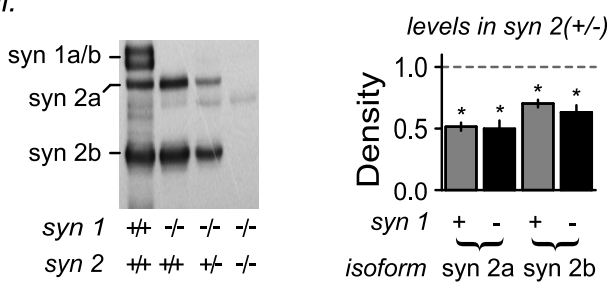

Figure 6. Induction of supply-rate depression. $A$, Plot of $w$ versus history of synaptic use. Synapses were stimulated with pairs of $20 \mathrm{~Hz}$ trains separated by fixed $20 \mathrm{~s}$ rest intervals as diagrammed (top). The length of the first train of each pair was varied experimentally, and subsequent RRP replenishment during the 20 s rest intervals was used to calculate $w$ in Equation 1 assuming $\tau_{f}=6.7 \mathrm{~s}$ and $\tau_{s}=55 \mathrm{~s}$. Plotted are fractional RRP replenishment $(y$-axis on left side) and $w$ (right side) versus the cumulative response during the first train [DKO, $n=17$ trials from 9 preparations; WT, $n=31$ trials from 17 preparations; WT data are a subset of the data set published previously in the study by Garcia-Perez et al. (2008, their Fig. 7)]. The dashed lines (green) are the working model with values for $r$ as indicated. The gray box labeled "single-point assay" contains the recovery values after trains of 120 pulses. The $x$-axis at the top is the cumulative release recalibrated into the number of RRP equivalents, which was calculated directly from the working model. $\boldsymbol{B}$, Synapsin dosage dependence of induction of supply-rate depression. $\boldsymbol{B i}$, Recovery during $20 \mathrm{~s}$ rest intervals following 120 pulse trains for the indicated genotypes ( $n \geq 10$ trials from 6 preparations; ${ }^{* *} p<0.01$ compared to WT; ${ }^{\# \#} p<0.01$ compared to synapsin 2 heterozygote). Recovery was measured as the size of the aggregate response during the second train divided by the size of the aggregate response to the first 60 pulses of the first train, which is simpler than the fractional RRP replenishment measurements plotted in $\boldsymbol{A}$ (see Materials and Methods). Bii, Western blot analysis of synapsin 2 levels in hippocampal tissue homogenates vious studies where no alterations in RRP replenishment time courses were detected because the previous studies examined replenishment after expending only the initial RRP contents. Indeed, 20-s-long rest intervals were sufficient for almost complete RRP replenishment at both DKO and WT synapses after shorter trains of only 20 action potentials (Fig. $6 \mathrm{~A}$, leftmost data points). (This was additionally observed in osmotic shock experiments similar to those in Fig. 7A; data not shown).

\section{Intact activity-dependent acceleration of vesicle trafficking}

Together, the results seem contrary to the concept that synapsins are involved in activity-dependent acceleration of vesicle trafficking (Greengard et al., 1993; Jensen et al., 2007). First, the invariant time constants in Equation 1 suggest that the mechanisms underlying RRP supply operate with the same bimodal kinetics at both DKO and WT synapses, whereas knocking out an acceleration mechanism would make one of the modes slower, which was not seen. Second, the activity-dependent acceleration that has been observed at WT Schaffer collateral synapses is saturated by the first 10 action potentials (Stevens and Wesseling, 1998; Wesseling and Lo, 2002), whereas defects in short-term plasticity at DKO synapses were not detected until after $\sim 60$ pulses of stimulation (Fig. $3 B$ ). To confirm the normal operation of the acceleration mechanism at DKO synapses, additional experiments were conducted on autapses using a previously designed protocol (Stevens and Wesseling, 1998) that again involved combining electrical stimulation with pairs of osmotic challenges for measuring the amount of RRP replenishment over fixed rest intervals (Fig. 7B, top). Consistent with an intact acceleration mechanism, 10 action potentials produced robust acceleration (Fig. $7 B$ ) in three of three autapses, which was similar in magnitude $(2.1 \pm$ 0.17 -fold; $p<0.02$ ) to the acceleration seen previously in WT synapses (Stevens and Wesseling, 1998, their Fig. 8).

\section{No change in release probability}

Because the timing of induction of supply-rate depression is thought to depend on the amount of neurotransmitter released (Stevens and Wesseling, 1999a), the earlier induction at DKO synapses suggests that synapsins are involved in determining the relationship between release and induction. An alternative explanation, however, would be that the DKO phenotype could indirectly result from hidden enhancement of the baseline level of release because synapses that initially released more neurotransmitter might depress earlier, for example, as a consequence of exhausting reserve pools more rapidly. To test this, we compared the time course of MK801 blockade of NMDA receptor responses during low-frequency stimulation at DKO and WT synapses in slices (Hessler et al., 1993). No difference was detected (Fig. 8 A), indicating that the initial probability of release was not enhanced (Manabe and Nicoll, 1994). Together with the observation that the release of transmitter depresses more quickly than WT during heavy stimulation (Fig. 3B), these results confirm that DKO synapses cumulatively release less neurotransmitter than WT during comparable amounts of heavy use, implicating synapsins in determining the relationship between release and induction of quantification, optical density of synapsin 2 bands from synapsin 2 heterozygotes was normalized by the density of the corresponding bands from animals with both alleles $\left({ }^{*} p<0.05 ; n=\right.$ 3 for $\operatorname{syn} 1(+/+)$ background, $n=6$ for syn $1(-/-)$ background). Error bars indicate SEM. 
A
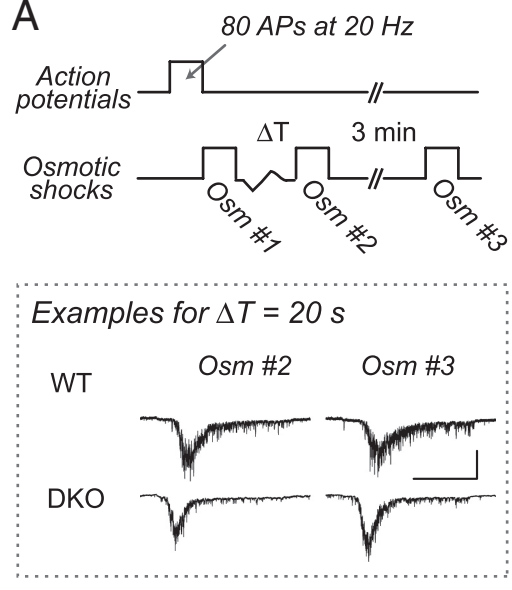

Full time course (DKO)

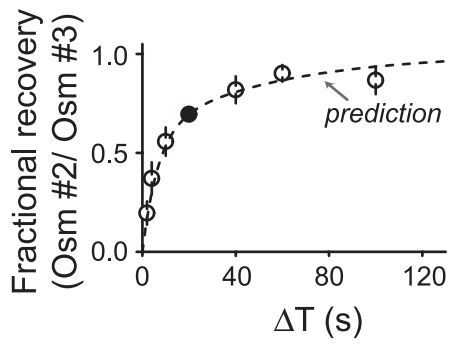

B
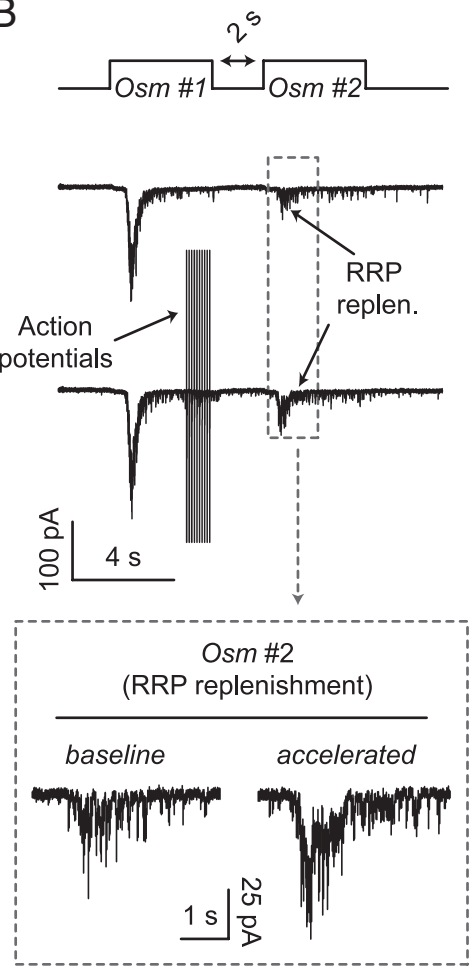

Figure 7. Osmotic shock experiments in autapses. A, Confirmation of earlier onset of supply-rate depression. RRP replenishment was measured at cell culture autapses following 80 presynaptic action potentials as diagrammed at top. The first osmotic shock $(0 \mathrm{sm})$ was used to ensure RRP depletion, the second was used to measure RRP replenishment over the intervening rest interval, and the third, 3 min later, was used to estimate the fully replenished RRP size in a way that avoids erroneously ascribing rundown to incomplete RRP replenishment (Stevens and Wesseling, 1999a). Box, Example responses to the second and third osmotic shocks when $\Delta T=20 \mathrm{~s}$. Calibration: WT, 2s, 50 pA; DK0, $2 \mathrm{~s}, 200 \mathrm{pA}$. The experiment was designed to recapitulate results for the single-point assay used in Figure $6 B$ and the difference between DKO and WT matched predictions; see Results for quantification. Bottom, Full time course for DKO synapses. The filled circle is the DKO data from the trials where $\Delta T=20 \mathrm{~s}(n=6)$, used to extrapolate the value of $w$ in Equation 1 (i.e., $w=0.60 ; n \geq 3$ for each data point). The dashed line is the resulting prediction generated by Equation 1 . All recovery values were adjusted for the $5 \%$ rundown that typically occurs over the 3 min between the second and third osmotic shocks (Stevens and Wesseling, 1999a); see Stevens and Wesseling (1999a) for WT RRP replenishment time courses measured with osmotic shocks. B, Preservation of activity-dependent acceleration of vesicle trafficking. RRP replenishment during 2-s-long rest intervals was measured at fully rested DKO synapses with pairs of osmotic shocks as diagrammed at the top, without (baseline) and with (accelerated) 10 action potentials during the final $1 \mathrm{~s}$ of the first shock. Example traces are plotted; see Results for quantification. The dashed gray boxes contain the responses elicited by the second shocks of each pair, showing acceleration.

supply-rate depression. See Figure 10 for confirmation of this result with FM1-43 in cell culture.

\section{No change in enhancement: paired-pulse facilitation and augmentation}

To test further the specificity of the DKO phenotype for the induction of supply-rate depression, we additionally measured elements of short-term enhancement, which are caused by residual $\mathrm{Ca}^{2+}$-dependent increases in the efficiency of the release machinery and seem to operate independently of the vesicle-trafficking mechanisms assayed above (Stevens and Wesseling, 1999b; Garcia-Perez and Wesseling, 2008). Although short-term depression dominates under our experimental conditions, facilitation, with a decay lasting hundreds of milliseconds, and augmentation, lasting tens of seconds, are operant but masked during much of the stimulation protocols used here (Garcia-Perez and Wesseling, 2008). Facilitation can be at least partially characterized as the paired-pulse ratio at the beginning of repetitive trains, but was not significantly different at DKO versus WT synapses in slices (Fig. 8B). The characteristic parameters of augmentation were extracted from the experiments in Figure 5 using a recently

developed analytical procedure (GarciaPerez and Wesseling, 2008), but both the amplitude and time course of decay were similar at DKO and WT synapses (Fig. $8 C)$. Together, these results suggest that short-term enhancement is not substantially affected by knocking out both synapsin 1 and 2. Nevertheless, Figure $8 B$ shows that single knock-outs exhibit altered paired-pulse ratios, which is in agreement with the original analysis as is the disappearance of this aspect of the phenotype in DKO (Rosahl et al., 1993, 1995).

\section{Single parameter permutation}

We then tested the working model against the complete set of DKO results and found that changing the value of the single parameter $r$ from 4.0 to 2.6 could account for all of the observed differences (Figs. 3, 6A, green lines). The three rate parameters showed no change, as was expected, because $\alpha$ and $\gamma$ determine $\tau_{f}$ and $\tau_{s}$, which were not different from WT (Fig. 5B), and $\alpha$ and $\beta$ set short-term plasticity characteristics at the beginning of repetitive stimulation, which were likewise not different from WT (Fig. $3 A$ ). A key result that distinguished the working model from competing alternatives was the third of the three initial constraints that the recovery time course of the aggregate response to the second of a pair of extended stimulus trains followed the single exponential characterized by $\tau=\tau_{s}$ from Equation 1 (Fig. $1 C$ ), and this property was retained by DKO synapses (Fig. 9A). Remarkably, even the modest activitydependent acceleration of $\gamma$ that was required for fitting WT results continued to be required for the DKO results (Fig. 9B), strongly supporting the hypothesis that the alteration in vesicle trafficking is limited to the mechanism described by $r$. In the context of the physical interpretation depicted in Figure $1 D$, the decrease in $r$ would imply that individual vesicle-tethering units would connect together an average of 2.6 vesicles in DKO compared to 4 in WT terminals, which would additionally explain the $\sim 35 \%$ reduction in vesicle number seen in DKO terminals (Rosahl et al., 1995) if the total number of docked and nondocked tethering units was unchanged.

We modeled the nonwhole number value for $r=2.6$ with the weighted average of models with $r=2$ and $r=3$ (see Materials and Methods). Although some more complicated weighting schemes could also work, extreme combinations such as weighted averages of $r=4$ and $r=1$ did not work because the simulations predicted a three-phase onset of supply-rate depression that includes an initial fast component followed by an intermediate plateau phase and then a slower decay component that is not seen in Figure 6A.

Reserve pool depletion mechanism for supply-rate depression In any case, Figure $1 D$ was not the only possible physical interpretation of the working model. A competing hypothesis has 
A

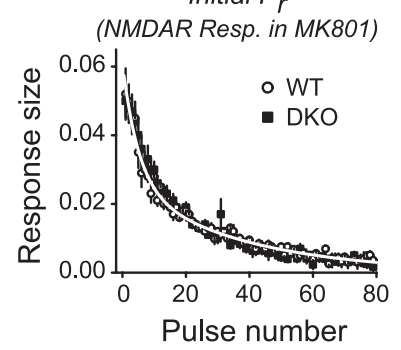

B i. First 20 responses

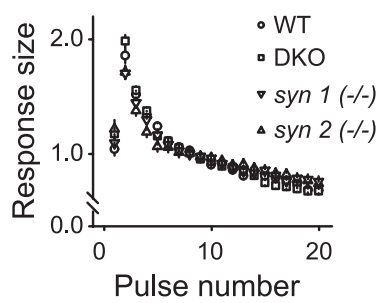

ii.

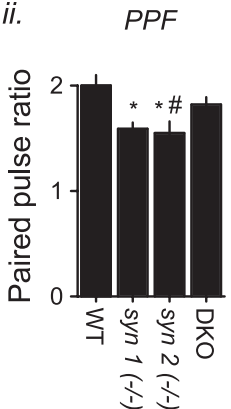

C

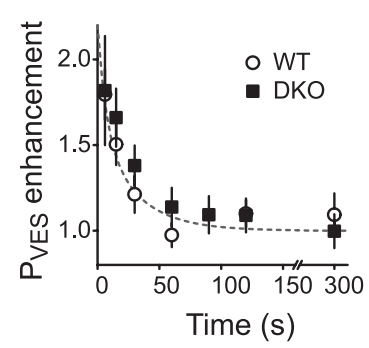

Figure 8. Unaltered release parameters. A, Initial release probability. Use-dependent blockade of the NMDA component of synaptic responses was monitored every $8 \mathrm{sin}$ the presence of MK801 $(n=10$ for DK0; $n=9$ for WT). Mean response sizes were normalized by the sum of all responses. The best-fitting double exponential for WT data (white line) had exponential components of $5 \pm$ 1 and $37 \pm 3$ responses, with $57 \pm 3 \%$ weighting for the faster exponential component. The same function fit the DKO data equally well $\left(R^{2}=79 \mathrm{vs} 78 \%\right)$, indicating that the initial release probability was similar. $\boldsymbol{B}$, Early short-term plasticity. Bi, Responses to 20 pulses at $20 \mathrm{~Hz}$, normalized by the mean size of all 20 responses $(n=66$ for WT, 20 for syn $1(-/-)$, 25 for syn $2(-/-)$, 55 for DKO). Bii, Paired-pulse ratio calculated as the size of the second response divided by the size of the first for each experiment before calculating the mean $\left({ }^{*} p<0.05\right.$ compared to WT; ${ }^{\#} p<$ 0.05 compared to DKO).C, Augmentation. Relative enhancement in the probability of release per readily releasable vesicle ( $P_{\mathrm{VES}} \mathrm{S}$ enhancement) during rest intervals following 600 -pulse trains were calculated as in Garcia-Perez and Wesseling (2008, their Fig. 4B), and the theoretical curve (dashed line) is identical to the one in their figure. Briefly, recovery from depression after each rest interval was measured in two ways from the responses elicited by a second stimulus train that was long enough to empty the RRP: RRP replenishment was estimated from the sum of all responses, as in Figure 5 , whereas the substantially faster recovery of the response elicited by single pulses was calculated from the first response. $P_{\text {VES }}$ enhancement for each time point was then calculated by dividing the single response recovery value by the RRP replenishment value. Error bars indicate SEM.

A
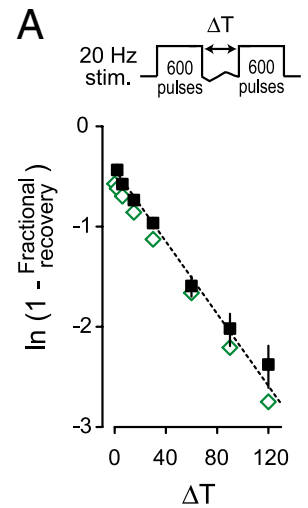

B
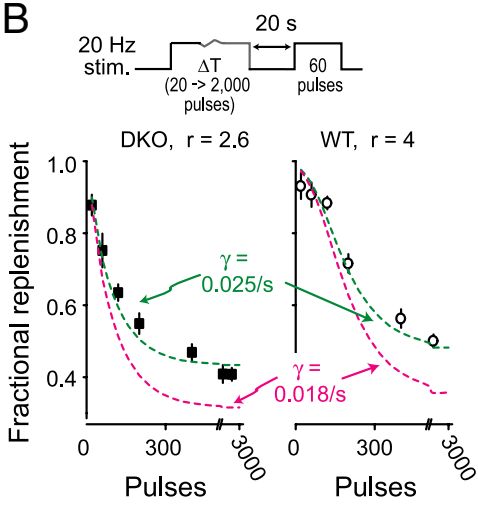

Figure 9. Specific additional tests of the working model at DKO synapses. $\boldsymbol{A}$, DKO synapses satisfy the third of the initial general constraints. The analogous result for WT is in Figure 1C. Plotted is the logarithm of 1 minus recovery of the sum of all responses during the second trains versus the intertrain interval ( $n=22$ trials from 29 preparations). The dashed line is the single exponential with $\tau=55 \mathrm{~s}$, which is the value of $\tau_{s}$ in Equation 1 that was extracted from Figure 5 for both WT and DKO. Green diamonds are simulation results using the working model with $r=2.6$ and $\gamma=0.018 /$ s. (i.e., $1 / 55 \mathrm{~s}$ ). $\boldsymbol{B}$, Replot of data in Figure $6 A$, except with the number of pulses of stimulation on the $x$-axis, showing that the working model with $\gamma=0.018 / \mathrm{s}$ (magenta) during activity does not fit results for either DKO or WT synapses, whereas $\gamma=$ $0.025 / \mathrm{s}$ (green) does fit in both cases.

alternatively ascribed supply-rate depression to use-dependent inhibition of the docking and priming machinery in the active zone (Stevens and Wesseling, 1999a), which would additionally explain why the majority of vesicles within presynaptic terminals do not participate in the exocytic/endocytic cycle even during heavy drive (Harata et al., 2001). However, failure of many vesicles to recycle does not necessarily rule out pool depletion ideas because the presence of nonparticipating vesicles can be explained as an immobile reserve pool (Rizzoli and Betz, 2005).

Also consistent with the inhibition hypothesis, however, is the routine observation that a substantial amount of FM1-43 is trapped inside synaptic terminals when used to stain recycling vesicles (Ribchester et al., 1994; Ryan and Smith, 1995). The mechanism remains unknown, but the inhibition hypothesis would seem to provide a natural explanation because it would

predict that some vesicles containing FM1-43 would be blocked from undergoing exocytosis as a consequence of activation of the hypothetical inhibitory mechanism. We thus reasoned that since supply-rate depression has an earlier onset at DKO synapses, the inhibition hypothesis would predict that vesicle trapping would begin earlier and consequently apply to a greater fraction of the recycled vesicles.

In experiments designed to address this, FM1-43 staining in conventional cell cultures was 33\% less at DKO compared to WT synapses (Fig. 10). Subsequent destaining was likewise less, and the rate of destaining was slightly faster (data not shown; but see Gitler et al., 2008). Considered on their own, these results are consistent both with inhibition and with pool depletion hypotheses. However, the level of residual FM1-43 staining left over after the destaining was less at DKO synapses, and, as a proportional quantity, the ratio of the residual staining to the level before destaining was not greater $(0.26 \pm 0.02$ for $\mathrm{DKO}$ vs $0.33 \pm 0.02$ for WT). Although it is not known whether the residual FM1-43 was trapped in synaptic vesicles or in some other structure (Ribchester et al., 1994), the result argues against the inhibition hypothesis in either case. If the FM1-43 were trapped in vesicles, the trapping would occur proportionally for DKO and WT, whereas inhibition predicts more trapping at DKO synapses, and the increase would have been detectable in the experiments of Figure 10 (calculations not shown). On the other hand, if the FM1-43 were instead trapped in some other structure, the result implies that vesicular trapping does not occur at either DKO or WT synapses, which would be contrary to predictions of the inhibition hypothesis that a substantial amount of vesicle trapping would occur.

\section{Discussion}

We have developed and tested a new theoretical model for ratelimiting steps in presynaptic vesicle trafficking at Schaffer collateral synapses. The new model departs from previous concepts that invoked gradual depletion of a reserve pool to account for the slow induction of deep forms of short-term depression, but a departure was needed because the mass-action rules at the heart of previous models are not consistent with several key observations. We additionally show that the phenotype of synapsin 

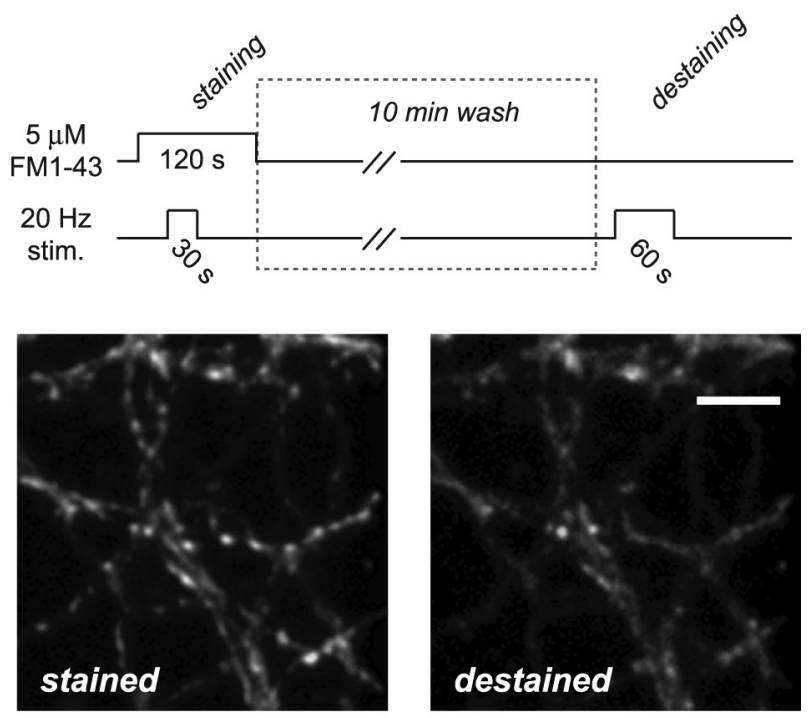

absolute values (during destaining)

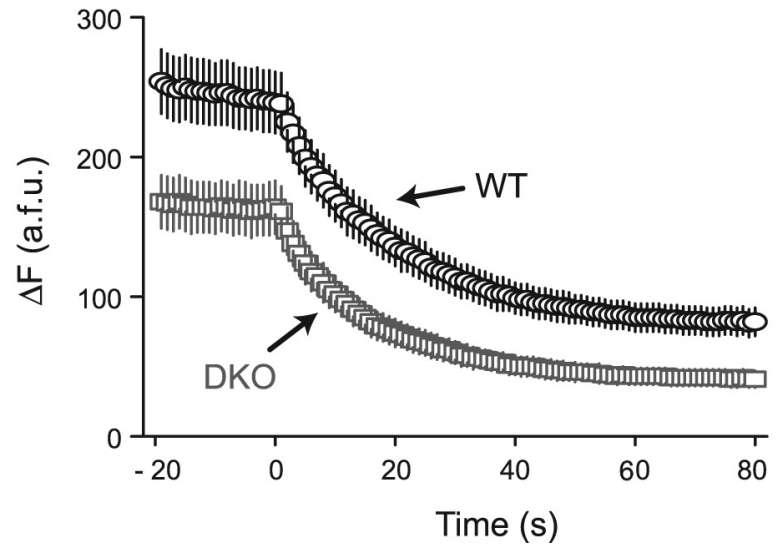

Figure 10. Less residual FM1-43 accumulation argues against increased trapping of recycling vesicles. DKO and WT synapses in cell culture were stained with FM1-43 using electrical field stimulation and subsequently destained, as diagramed at the top. Images are of DKO synapses after staining and after subsequent destaining. $\Delta$ Fversus time values are the mean of the median values after background subtraction starting 20 s before destaining and continuing for 20 s afterward ( $n=10$ fields for DK0; $n=15$ fields for WT, each containing 22-97 fluorescent punctae). Both the total amount and residual level of staining were significantly reduced in DKO $(p<0.01)$, and the residual level as a relative quantity was not increased (see Results).

knock-outs can be cleanly characterized by changing the value of only a single one of the model's four parameters.

The new model provides a quantitative framework for investigating the functional roles of molecular components operating upstream of the final fusion step, which have been difficult to assess. The analysis of the synapsin knock-outs is a case in point. The finding that the single parameter $r$ is selectively altered without requiring new parameters suggests that $r$ represents a ratelimiting quantity in the vesicle cycle and thus could be a target of physiological modulation. Modulation might occur via synapsin phosphorylation (Greengard et al., 1993; Chi et al., 2003), but further experiments will be needed to address this issue. The value of $r$ depended on the dosage of synapsin 2 but not synapsin 1, which is consistent with previous studies (Rosahl et al., 1995; Gitler et al., 2008).

The new model is mathematically simpler than previous models (Granseth and Lagnado, 2008), which reflects the simplicity of three overarching kinetic constraints on RRP supply that were extracted from previous and new experimental findings. Parsimony was not the main motivation for adopting the model, and indeed we are not aware of structurally distinct alternatives, even when more complexity is allowed.

\section{Comparison to release}

We emphasize that the model pertains to rate-limiting steps in vesicle trafficking that are upstream of the release machinery and is not meant to explain other important aspects of short-term plasticity (Wu and Betz, 1998; Trommershauser et al., 2003; Hennig et al., 2008; Pan and Zucker, 2009). Indeed, the large changes in synaptic strength that typically occur before the RRP has been exhausted and the fast rebound seen during subsequent rest intervals are largely caused by additional mechanisms such as RRP depletion (Wesseling and Lo, 2002), heterogeneity in release parameters between release sites (Trommershauser et al., 2003; Moulder and Mennerick, 2005), residual $\mathrm{Ca}^{2+}$-dependent enhancement of release (Delaney and Tank, 1994; Kalkstein and Magleby, 2004; Garcia-Perez and Wesseling, 2008), and other potential factors (Wadel et al., 2007; Hennig et al., 2008; Mochida et al., 2008).

\section{Comparison to other synapse types}

The new model applies to Schaffer collateral synapses, whereas measurements of rate-limiting vesicle-trafficking steps may not be feasible with similar techniques at some other types of synapses because of severe inactivation of $\mathrm{Ca}^{2+}$ channels that might effectively block release during heavy use (Hennig et al., 2008). Or, vesicle trafficking might follow more complicated rules at other synapse types (Richards et al., 2003; Crowley et al., 2007; Coleman et al., 2008; Sakaba, 2008). Nevertheless, Schaffer collateral synapses are similar to the majority of excitatory synapses throughout the brain, both at the level of electron microscopy (Peters and Palay, 1996) and in that they are typically driven sparingly during ordinary use (Ranck, 1973), but when driven extensively they exhibit substantial short-term depression (Chance et al., 1998).

A new reserve pool depletion model of supply-rate depression Although designed with the ultimate goal of evaluating the new theoretical model, the analysis of synapsin knock-outs additionally yielded a series of new intermediate findings that are valid independently. The increased depression at DKO Schaffer collateral synapses is caused by an alteration in vesicle trafficking at a stage that is upstream of release, which was previously in doubt. However, the baseline vesicle-trafficking rate is unchanged, and the acceleration that occurs during bursts of activity is preserved. Instead, the increased depression is caused by earlier induction of the slowest-to-recover component of short-term depression, which was previously termed "supply-rate depression" because it arises from depression in the rate at which vesicles are supplied to the RRP.

Two mutually exclusive types of mechanisms have been proposed to explain supply-rate depression. Reserve pool depletion models have been prevalent (Birks and MacIntosh, 1961; Elmqvist and Quastel, 1965; Richards et al., 2003; Granseth and Lagnado, 2008), but the mass-action assumption at their core is not consistent with kinetic measurements. However, alternatives where vesicle trafficking is inhibited without reserve pool depletion seem to be ruled out here. This leaves reserve pool depletion models where the supply-rate is not driven by mass action, such as the one depicted in Figure $1 D$. 
Figure $1 D$ is probably not the only possibility that is compatible with both the new theoretical model and the information in Figure 10, but it does already have some intriguing morphological support. For example, cytoskeletal structures with the correct length that might be vesicle-tethering units have been observed in Schaffer collateral presynaptic terminals in recent tomography studies (Siksou et al., 2007). In addition, much larger, but perhaps functionally analogous ribbons and T-bars may play a related role in more specialized synapse types (Owald and Sigrist, 2009).

\section{Synapsin hypothesis}

A much discussed synapsin hypothesis has been that synapsins regulate depression by setting the mobilization rates of reserve vesicles or the size of a reserve pool by cross-linking vesicles to the cytoskeleton (Greengard et al., 1993). However, the mobility of synaptic vesicles was not altered in the knock-outs (Gaffield and Betz, 2007), and both the putative immobile reserve and recycling reserve pools (Rizzoli and Betz, 2005) were decreased by similar amounts (Pieribone et al., 1995; Rosahl et al., 1995; Bogen et al., 2006), contrary to the expectation that only one of the two pools would be affected. In addition, our own attempts to incorporate into kinetic models the idea that synapsin phosphorylation accelerates vesicle trafficking have not been successful because the idea ultimately invokes mass-action rules to explain the speed of vesicle trafficking. Intriguingly, the interpretation in Figure $1 \mathrm{D}$ does implement the idea that synapsins are involved in determining reserve pool size and can therefore be thought of as a refinement of the synapsin hypothesis, although not precisely as originally formulated. If so, the value for $r$ of 2.6 at DKO synapses would indicate that tethering units, although shorter, would continue to be present in the absence of synapsins. This is consistent with the tomography study by Siksou et al. (2007), where the cytoskeletal structures linking vesicles together were seen in synapsin knockouts, although whether the structures are shorter in the knockouts remains to be determined.

\section{Rate-limiting role for endocytosis?}

The scheme in Figure $1 D$ does not include a rate-limiting role for endocytosis or a route for rereleasing vesicles shortly after endocytosis, which might be surprising given the recent interest in the physiological role of fast kiss-and-run endocytosis and other reports (Pyle et al., 2000; Granseth and Lagnado, 2008). However, although a rate-limiting role for endocytosis cannot be ruled out, available kinetic evidence argues against it. The parameter $\gamma$ seems to be too slow and is less dependent on temperature than endocytosis (Klyachko and Stevens, 2006; Garcia-Perez et al., 2008; Granseth and Lagnado, 2008). In addition, $\gamma$ does not become slower during heavy use, whereas endocytosis seems to (Sankaranarayanan and Ryan, 2000). Meanwhile, $\alpha$ seems to be too fast for classical endocytosis and too slow for kiss-and-run endocytosis (but see Zhang et al., 2009). Moreover, ascribing $\alpha$ to endocytosis would predict that newly endocytosed vesicles would have priority for release ahead of yet-to-be-released vesicles remaining in the recycling pool, which seems contrary to some observations (Ryan et al., 1993). On the other hand, an intriguing hypothesis that could be consistent with the working model would be that kiss-and-run vesicles become reavailable for release so quickly that the whole cycle would occur without being detected as rate-limiting in our experiments (Pyle et al., 2000). However, this hypothesis would imply that some of the neurotransmission quanta attributed to release of the RRP would instead result from release of quickly recycled vesicles, which would predict that the number of quanta of neurotransmitter available for release from within the RRP would be greater than the number of docked and primed vesicles (but see Schikorski and Stevens, 2001).

\section{Summary}

In summary, we have developed a new kinetic framework for identifying molecules and mechanisms that play rate-limiting roles in synaptic vesicle trafficking. In testing the framework, we identified a discrete deficit in synapsin DKO synapses where the value of only a single one of four parameters is altered. Importantly, the framework avoids the concept at the heart of previous models that vesicle-trafficking rates depend on the number of reserve vesicles, but the more general idea that the longest-lasting component of depression is caused by depletion of reserve stores needed to be preserved. Together, the results support a new cell biological scheme where local reserve pools consisting of four vesicles at WT synapses, and fewer at DKO synapses, are tethered to individual release sites and are replaced spontaneously as complete units about once per minute.

\section{Notes}

Supplemental material for this article is available at http://www.cima.es/ admin/docs_eventos/1306321563.pdf. The single pdf file contains four appendices detailing: (A) the derivation of Equation 1; (B) the origin of values in Figure $2 A ;(C)$ the reasoning against inhibition models ruled out by Figure 10; and (D) an example of why mass-action models are incompatible with Equation 1. This material has not been peer reviewed.

\section{References}

Birks RI, MacIntosh FC (1961) Acetylcholine metabolism of a synpathetic ganglion. Can J Biochem Physiol 39:787-827.

Bogen IL, Boulland JL, Mariussen E, Wright MS, Fonnum F, Kao HT, Walaas SI (2006) Absence of synapsin I and II is accompanied by decreases in vesicular transport of specific neurotransmitters. J Neurochem 96:1458-1466.

Burrone J, Li Z, Murthy VN (2006) Studying vesicle cycling in presynaptic terminals using the genetically encoded probe synaptopHluorin. Nat Protoc 1:2970-2978.

Chance FS, Nelson SB, Abbott LF (1998) Synaptic depression and the temporal response characteristics of V1 cells. J Neurosci 18:4785-4799.

Chi P, Greengard P, Ryan TA (2003) Synaptic vesicle mobilization is regulated by distinct synapsin I phosphorylation pathways at different frequencies. Neuron 38:69-78.

Coleman WL, Bill CA, Simsek-Duran F, Lonart G, Samigullin D, Bykhovskaia M (2008) Synapsin II and calcium regulate vesicle docking and the cross-talk between vesicle pools at the mouse motor terminals. J Physiol 586:4649-4673.

Crowley JJ, Carter AG, Regehr WG (2007) Fast vesicle replenishment and rapid recovery from desensitization at a single synaptic release site. J Neurosci 27:5448-5460.

Delaney KR, Tank DW (1994) A quantitative measurement of the dependence of short-term synaptic enhancement on presynaptic residual calcium. J Neurosci 14:5885-5902.

Elmqvist D, Quastel DM (1965) A quantitative study of end-plate potentials in isolated human muscle. J Physiol 178:505-529.

Gaffield MA, Betz WJ (2007) Synaptic vesicle mobility in mouse motor nerve terminals with and without synapsin. J Neurosci 27:13691-13700.

Garcia-Perez E, Wesseling JF (2008) Augmentation controls the fast rebound from depression at excitatory hippocampal synapses. J Neurophysiol 99:1770-1786.

Garcia-Perez E, Lo DC, Wesseling JF (2008) Kinetic isolation of a slowly recovering component of short-term depression during exhaustive use at excitatory hippocampal synapses. J Neurophysiol 100:781-795.

Gitler D, Cheng Q, Greengard P, Augustine GJ (2008) Synapsin IIa controls the reserve pool of glutamatergic synaptic vesicles. J Neurosci 28:10835-10843.

Granseth B, Lagnado L (2008) The role of endocytosis in regulating the strength of hippocampal synapses. J Physiol 586:5969-5982. 
Greengard P, Valtorta F, Czernik AJ, Benfenati F (1993) Synaptic vesicle phosphoproteins and regulation of synaptic function. Science 259:780-785.

Harata N, Pyle JL, Aravanis AM, Mozhayeva M, Kavalali ET, Tsien RW (2001) Limited numbers of recycling vesicles in small CNS nerve terminals: implications for neural signaling and vesicular cycling. Trends Neurosci 24:637-643.

Hennig MH, Postlethwaite M, Forsythe ID, Graham BP (2008) Interactions between multiple sources of short-term plasticity during evoked and spontaneous activity at the rat calyx of Held. J Physiol 586:3129-3146.

Hessler NA, Shirke AM, Malinow R (1993) The probability of transmitter release at a mammalian central synapse. Nature 366:569-572.

Huttner WB, Schiebler W, Greengard P, De Camilli P (1983) Synapsin I (protein I), a nerve terminal-specific phosphoprotein. III. Its association with synaptic vesicles studied in a highly purified synaptic vesicle preparation. J Cell Biol 96:1374-1388.

Jensen V, Walaas SI, Hilfiker S, Ruiz A, Hvalby O (2007) A delayed response enhancement during hippocampal presynaptic plasticity in mice. J Physiol 583:129-143.

Kalkstein JM, Magleby KL (2004) Augmentation increases vesicular release probability in the presence of masking depression at the frog neuromuscular junction. J Neurosci 24:11391-11403.

Klyachko VA, Stevens CF (2006) Temperature-dependent shift of balance among the components of short-term plasticity in hippocampal synapses. J Neurosci 26:6945-6957.

Lou X, Scheuss V, Schneggenburger R (2005) Allosteric modulation of the presynaptic Ca2+ sensor for vesicle fusion. Nature 435:497-501.

Manabe T, Nicoll RA (1994) Long-term potentiation: evidence against an increase in transmitter release probability in the CA1 region of the hippocampus. Science 265:1888-1892.

Meyn SP, Tweedie RL (2009) Markov chains and stochastic stability, Ed 2. Cambridge: Cambridge UP.

Mochida S, Few AP, Scheuer T, Catterall WA (2008) Regulation of presynaptic $\mathrm{Ca}(\mathrm{V}) 2.1$ channels by $\mathrm{Ca} 2+$ sensor proteins mediates short-term synaptic plasticity. Neuron 57:210-216.

Moulder KL, Mennerick S (2005) Reluctant vesicles contribute to the total readily releasable pool in glutamatergic hippocampal neurons. J Neurosci 25:3842-3850.

Owald D, Sigrist SJ (2009) Assembling the presynaptic active zone. Curr Opin Neurobiol 19:311-318.

Pan B, Zucker RS (2009) A general model of synaptic transmission and short-term plasticity. Neuron 62:539-554.

Peters A, Palay SL (1996) The morphology of synapses. J Neurocytol 25:687-700.

Pieribone VA, Shupliakov O, Brodin L, Hilfiker-Rothenfluh S, Czernik AJ, Greengard P (1995) Distinct pools of synaptic vesicles in neurotransmitter release. Nature 375:493-497.

Pyle JL, Kavalali ET, Piedras-Renteria ES, Tsien RW (2000) Rapid reuse of readily releasable pool vesicles at hippocampal synapses. Neuron 28:221-231.

Ranck JB Jr (1973) Studies on single neurons in dorsal hippocampal formation and septum in unrestrained rats. I. Behavioral correlates and firing repertoires. Exp Neurol 41:462-531.

Ribchester RR, Mao F, Betz WJ (1994) Optical measurements of activitydependent membrane recycling in motor nerve terminals of mammalian skeletal muscle. Proc Biol Sci 255:61-66.

Richards DA, Guatimosim C, Rizzoli SO, Betz WJ (2003) Synaptic vesicle pools at the frog neuromuscular junction. Neuron 39:529-541.

Rizzoli SO, Betz WJ (2005) Synaptic vesicle pools. Nat Rev Neurosci 6:57-69.

Roberts AC, Diez-Garcia J, Rodriguiz RM, Lopez IP, Lujan R, MartinezTurrillas R, Pico E, Henson MA, Bernardo DR, Jarrett TM, Clendeninn DJ, Lopez-Mascaraque L, Feng G, Lo DC, Wesseling JF, Wetsel WC, Philpot BD, Perez-Otano I (2009) Downregulation of NR3A-containing NMDARs is required for synapse maturation and memory consolidation. Neuron 63:342-356.
Rosahl TW, Geppert M, Spillane D, Herz J, Hammer RE, Malenka RC, Sudhof TC (1993) Short-term synaptic plasticity is altered in mice lacking synapsin I. Cell 75:661-670.

Rosahl TW, Spillane D, Missler M, Herz J, Selig DK, Wolff JR, Hammer RE, Malenka RC, Sudhof TC (1995) Essential functions of synapsins I and II in synaptic vesicle regulation. Nature 375:488-493.

Rosenmund C, Stevens CF (1996) Definition of the readily releasable pool of vesicles at hippocampal synapses. Neuron 16:1197-1207.

Ryan TA, Smith SJ (1995) Vesicle pool mobilization during action potential firing at hippocampal synapses. Neuron 14:983-989.

Ryan TA, Reuter H, Wendland B, Schweizer FE, Tsien RW, Smith SJ (1993) The kinetics of synaptic vesicle recycling measured at single presynaptic boutons. Neuron 11:713-724.

Sakaba T (2008) Two Ca(2+)-dependent steps controlling synaptic vesicle fusion and replenishment at the cerebellar basket cell terminal. Neuron 57:406-419.

Sankaranarayanan S, Ryan TA (2000) Real-time measurements of vesicleSNARE recycling in synapses of the central nervous system. Nat Cell Biol 2:197-204.

Schikorski T, Stevens CF (1997) Quantitative ultrastructural analysis of hippocampal excitatory synapses. J Neurosci 17:5858-5867.

Schikorski T, Stevens CF (2001) Morphological correlates of functionally defined synaptic vesicle populations. Nat Neurosci 4:391-395.

Schneggenburger R, Neher E (2005) Presynaptic calcium and control of vesicle fusion. Curr Opin Neurobiol 15:266-274.

Siksou L, Rostaing P, Lechaire JP, Boudier T, Ohtsuka T, Fejtova A, Kao HT, Greengard P, Gundelfinger ED, Triller A, Marty S (2007) Threedimensional architecture of presynaptic terminal cytomatrix. J Neurosci 27:6868-6877.

Stevens CF, Wesseling JF (1998) Activity-dependent modulation of the rate at which synaptic vesicles become available to undergo exocytosis. Neuron 21:415-424.

Stevens CF, Wesseling JF (1999a) Identification of a novel process limiting the rate of synaptic vesicle cycling at hippocampal synapses. Neuron 24:1017-1028.

Stevens CF, Wesseling JF (1999b) Augmentation is a potentiation of the exocytotic process. Neuron 22:139-146.

Sun J, Bronk P, Liu X, Han W, Sudhof TC (2006) Synapsins regulate usedependent synaptic plasticity in the calyx of Held by a Ca2+/calmodulindependent pathway. Proc Natl Acad Sci U S A 103:2880-2885.

Sun J, Pang ZP, Qin D, Fahim AT, Adachi R, Sudhof TC (2007) A dual$\mathrm{Ca} 2+$-sensor model for neurotransmitter release in a central synapse. Nature 450:676-682.

Trommershauser J, Schneggenburger R, Zippelius A, Neher E (2003) Heterogeneous presynaptic release probabilities: functional relevance for short-term plasticity. Biophys J 84:1563-1579.

Tsodyks MV, Markram H (1997) The neural code between neocortical pyramidal neurons depends on neurotransmitter release probability. Proc Natl Acad Sci U S A 94:719-723.

Wadel K, Neher E, Sakaba T (2007) The coupling between synaptic vesicles and $\mathrm{Ca}(2+)$ channels determines fast neurotransmitter release. Neuron 53:563-575

Wesseling JF, Lo DC (2002) Limit on the role of activity in controlling the release-ready supply of synaptic vesicles. J Neurosci 22:9708-9720.

Wu LG, Betz WJ (1998) Kinetics of synaptic depression and vesicle recycling after tetanic stimulation of frog motor nerve terminals. Biophys J 74:3003-3009.

Wu LG, Borst JG (1999) The reduced release probability of releasable vesicles during recovery from short-term synaptic depression. Neuron 23:821-832.

Zampighi GA, Fain N, Zampighi LM, Cantele F, Lanzavecchia S, Wright EM (2008) Conical electron tomography of a chemical synapse: polyhedral cages dock vesicles to the active zone. J Neurosci 28:4151-4160.

Zhang Q, Li Y, Tsien RW (2009) The dynamic control of kiss-and-run and vesicular reuse probed with single nanoparticles. Science 323:1448-1453. 